\title{
Connettere il territorio tra patrimonio e informazione: banche dati e modelli per le Cultural Heritage Routes
}

\author{
Raffaella De Marco \\ Anna Dell'Amico
}

\section{Abstract}

II contributo affronta la 'connessione' tra patrimonio costruito e territorio approfondendo le forme ed i ruoli della 'rappresentazione' multidimensionale declinata tra 'architettura' e 'rotta culturale'. Le Cultural Heritage Routes [ICOMOS 2008] assumono un ruolo chiave come collettori di un patrimonio che supera i propri confini geografici e politici. Attraverso le esperienze offerte dal progetto H2020-RISE-PROMETHEUS (2019-202 I) viene condotta una riflessione sul patrimonio diffuso del bacino dell'Upper Kama, in Russia, quale caso pilota sulla rotta culturale dell'architettura religiosa ed insediativa del mercato del sale tra Europa e Asia.

II progetto è indirizzato all'elaborazione di una Charta programmatica per Modelli Informativi Territoriali replicabile in contesti europei. L'obiettivo è evidenziare i molteplici livelli di conoscenza stratificati sul singolo sito-monumento, dalla fabbrica costruttiva al complesso architettonico fino alla rete di relazione territoriale con il bacino culturale, in un processo di semantizzazione morfologica, tecnologica e tipologica che permetta di coordinarne la gestione combinata di memoria, informazione ed intervento sull'opera.

Parole chiave

patrimonio diffuso, rilievo digitale, sistemi informativi, Cultural Heritage Routes, Upper Kama.

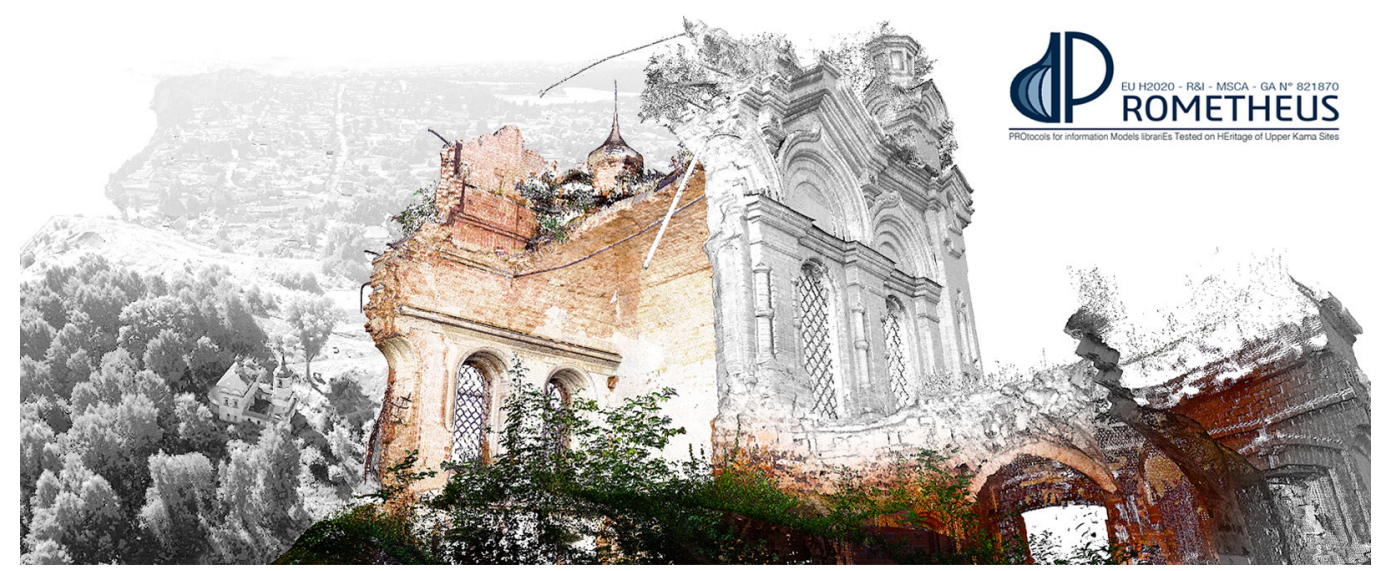




\section{Le Cultural Heritage Routes come scenario di connessione tra territorio e monumento}

Lo studio delle connessioni tra patrimonio costruito e territorio si fonda sull'analisi dell'impronta culturale di reciproca influenza tra sito e rete paesaggistica di appartenenza [I]. I caratteri riscontrati sono al tempo stesso tangibili, materialmente rappresentati dalle testimonianze costruttive ed infrastrutturali, ed intangibili, nella memoria di tracciati e reti socioeconomiche non più esistenti ma ancora influenzanti la localizzazione di monumenti e rotte. Tra flussi insediativi e stilistici improntati sulle connessioni 'di bacino', è riconosciuta con sempre maggiore enfasi una classificazione di immagini ed informazioni sul 'patrimonio diffuso' [2] generato dall'intreccio di crocevia geografici e culturali, dove la forma dell'intervento antropico sul paesaggio lascia ancora emergere una più profonda relazione tra siti e monumenti appartenente alla scala del territorio. II riconoscimento di reti estese di contaminazione tra tradizioni cosmopolite e territori locali, in particolare tra l'Europa e i continenti limitrofi, classifica scenari in grado di affermare e mantenere specifici caratteri identitari. La comprensione e strutturazione di tali caratteri si pone necessaria per operare una gestione e valorizzazione degli stessi, conformando il patrimonio locale tra memoria e pianificazione come specifico 'paesaggio culturale' [3].

II riconoscimento delle 'rotte culturali' [4], oggi al centro di programmi comunitari per la loro valorizzazione e gestione, ha sensibilizzato il patrimonio europeo condiviso sul dinamismo spaziale e temporale dei suoi 'bacini' architettonici, mettendo in evidenza i fenomeni storici di scambio e dialogo tra popoli e regioni con i quadri contemporanei di pianificazione e gestione del territorio. La continuità attraverso spazio e tempo diventa così la chiave di classificazione del Patrimonio Culturale lungo le Cultural Heritage Routes, definite come una categoria inclusiva tra patrimonio materiale e memoria territoriale in grado di comprendere valori tangibili ma dispersi dei processi insediativi. Le qualità connettive testimoniate dai caratteri visuali e costruttivi dell'architettura e del luogo sono ricercate e classificate come valori parametrici di sintesi e comprensione della cultura territoriale, conformando dinamicamente i paesaggi culturali nel tempo (fig. I).

Fig. I. Classificazione preliminare degli Object of Cultural Heritage $(\mathrm{OCH})$ per la gestione territoriaRoute dell'Upper Kama Route dell'Upper Kama: Monumento (Monument) Complesso (Ensemble) e Sito (Site) definiscono livelli di interazione e sviluppo di descrittori architettonici, paesaggistici ed infrastrutturali per l'analisi dei poli monumentali dell'Upper Kama.
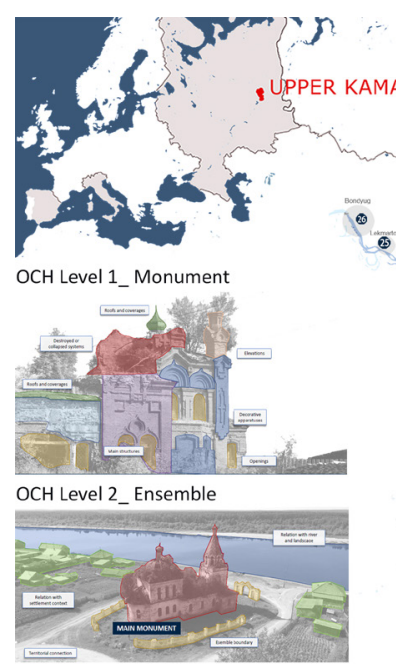

$\mathrm{OCH}$ Level 3_Site
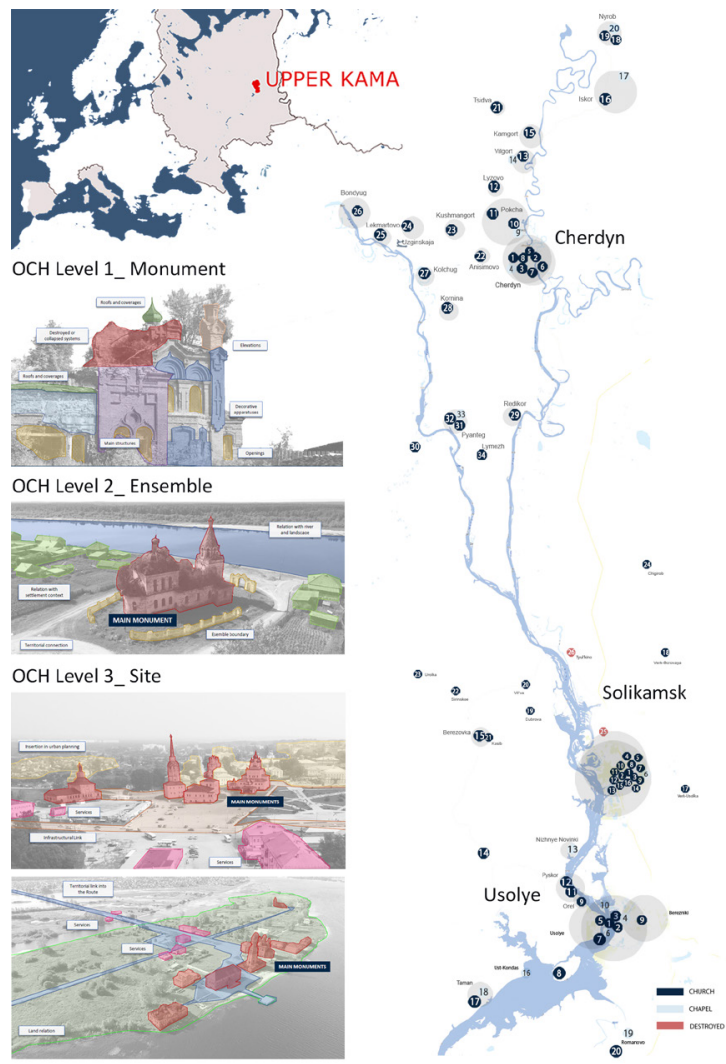
Alla rappresentazione è affidato il riconoscimento di caratteristiche e valori di connessione tra siti fisicamente distanti ma culturalmente uniti, supportata da ordini spaziali, gerarchie e geometrie di forme a loro volta connesse in una diretta derivazione da tecnologie, materiali e stili come elementi tangibili di carattere fisico dei siti e di identificazione tipologica della rotta. Pertanto, i confini geografici aiutano a visualizzare la Cultural Heritage Route, determinandone il percorso o lo sviluppo nel tempo, ma non possono contenerne il fenomeno culturale, assimilato dal metodo di conoscenza dei suoi caratteri ed esprimibile solamente con un processo di sintesi dei suoi contesti tipologici, quale quello operato attraverso il disegno.

Fig. 2. Siti monumentali dell'Upper Kama e relazione con il paesaggio: dall'alto da sinistra, Church of Saints Peter and Paul a Church Nyrob (1704). Chapel Nyrob (1704), Chapel of Kazan Mother of God Pokcha (1913), Church of Nativity a Iskor (1793), Church of St. Nicholas a Uzhginskay ( 1874), Church of Annunciation of the Blessed Virgin a Pokcha (1785). Church of Presentation of Blessed Virgin a Kamgort (1915) Church of the exaltation Church of the exaltatior of the Holy Cross a Bondjug (1858), Church of All Saints a Cherdyn (1817).
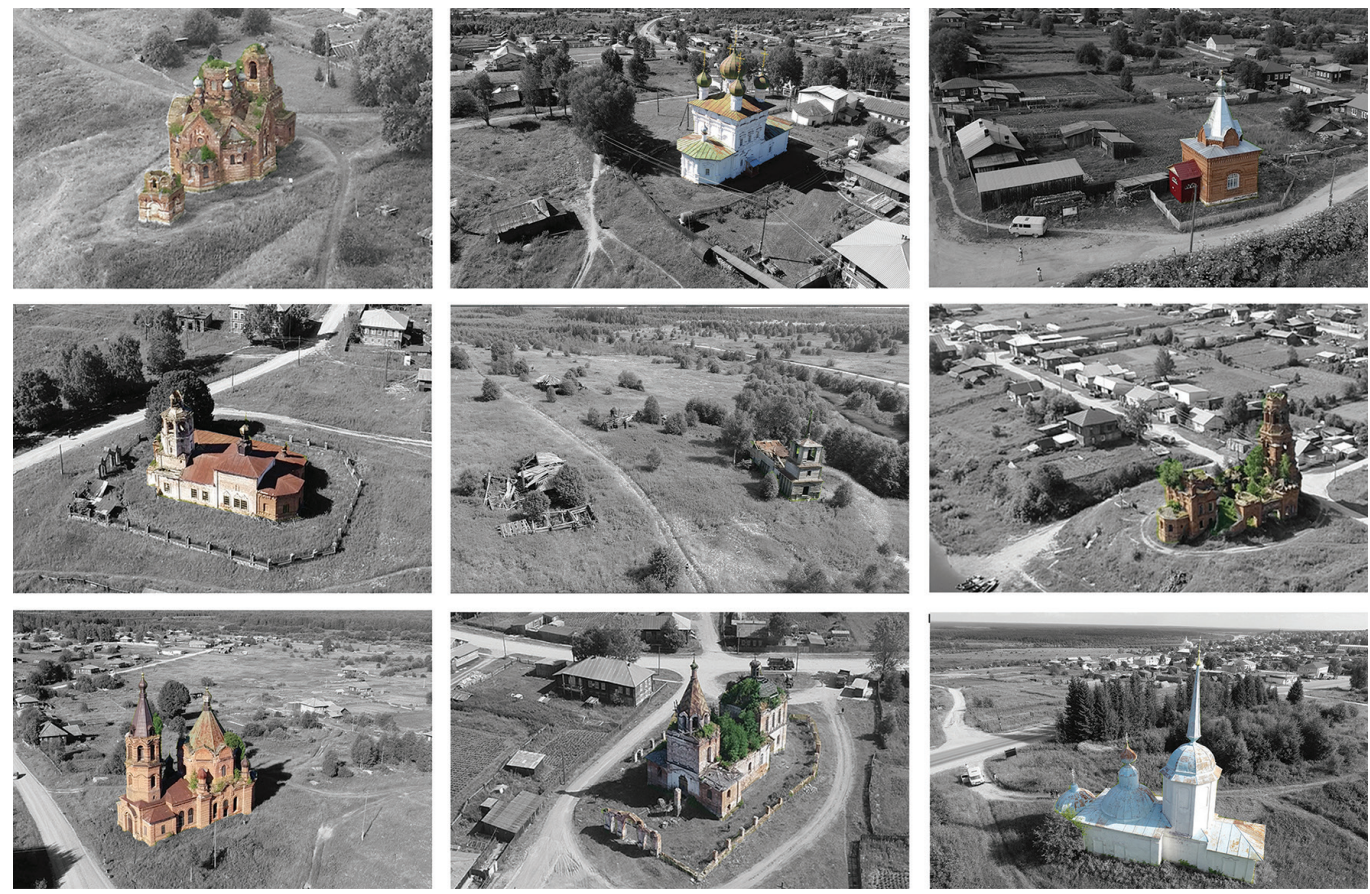

Analisi dei caratteri architettonici per la rappresentazione delle rotte culturali: il caso dell'Upper Kama, Russia

Il caso pilota dell'Upper Kama, già interessato da studi paesaggistici degli ultimi decenni [5] e da un'esperienza di ricerca internazionale pluriennale sulla documentazione digitale di alcuni complessi storici [6], è stato individuato come emblematica Cultural Heritage Route di convivenza tra architetture monumentali e criticità territoriali diffuse (fig. 2), riflettendo l'impronta stilistica e formale della cultura europea oltre i suoi confini politici. In tali termini, i suoi siti raccolgono un catalogo di caratteri architettonici, varietà stilistiche, soluzioni costruttive ed una molteplicità di materiali da costruzione, con relative patologie di degrado e conservazione, tali da caratterizzare l'identità del suo bacino e del corrispondente paesaggio culturale (fig. 3).

La regione dell'Upper Kama, sviluppata attorno al bacino del fiume Kama, si estende a nord del Krai di Perm per circa 7.000 kmq, a ovest dei monti Urali nella Russia europea. Le risorse minerarie e il ruolo di crocevia commerciale del periodo imperiale hanno portato l'Upper Kama ad un intenso sviluppo culturale ed architettonico tra il XV secolo, con i primi insediamenti industriali, e il XVIII secolo, fino al rapido declino con la Rivoluzione russa di ottobre (19/7). La scoperta delle saline locali da parte di famiglie mercantili, come gli Stroganov o i Golitsyns, ha decretato lo sviluppo di insediamenti urbani caratterizzati da monumentali complessi ortodossi, chiese, torri campanarie e cappelle familiari, richiamando architetti ed 
Fig. 3. L'identificazione e classificazione dei caratteri stilistici evidenzia forme e geometrie locali, sviluppate nei tradizionali motivi decorativi zuchcovi sekirovidnymi, oltre ad un accostamento di prevalenti materiali lignei din laterizio con relative patologie di degrado. tartare ed europee anche
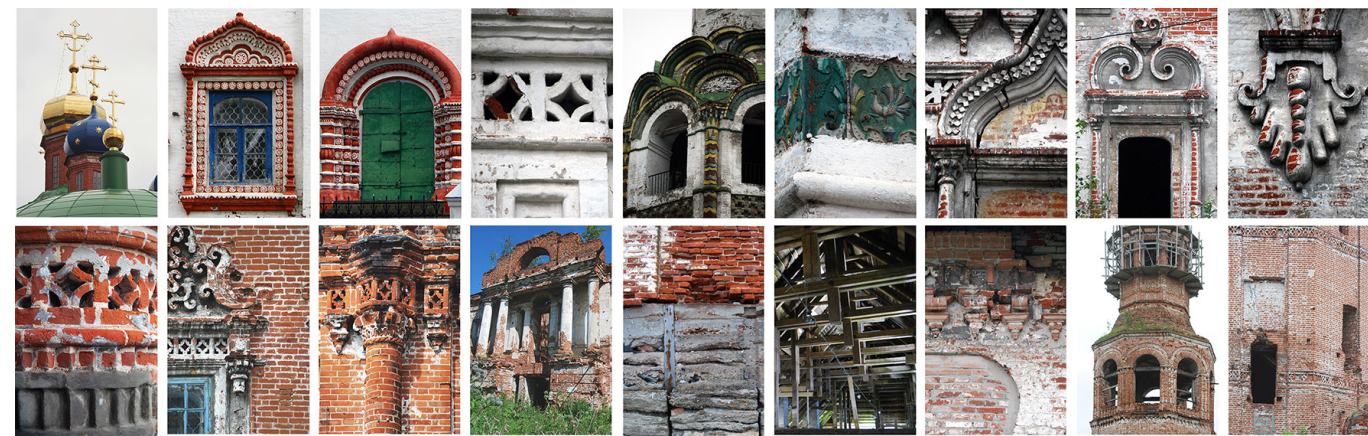

artisti continentali a definire un nuovo e dominante profilo architettonico nel paesaggio fluviale. II mercato del sale, che dall'Europa raggiunse gli Urali per commerciare con la Cina, portò alla diffusione di numerosi centri urbani ed all'impronta dispersiva degli appartenenti siti monumentali, associati ai distretti di Solikamsk (I 430), Cherdyn (I535) e Usolye (I 606) e tutt'oggi assegnati ad una frammentata amministrazione territoriale convogliata al controllo centrale del Perm Krai [7].

L'impronta insediativa, più stabilizzata dalle esigenze commerciali rispetto ai villaggi della campagna russa, ha determinato un fenomeno culturale di trasformazione dalla tipica architettura lignea a soluzioni costruttive in muratura di laterizio e pietra, congiuntamente a temi stilistici unitari di forma e decoro derivati dal barocco moscovita e dalle influenze europee. I siti dell'Upper Kama sono caratterizzati da architetture religiose monumentali e spesso isolate, originariamente appartenenti a complessi più estesi di carattere cerimoniale o dedicati al pellegrinaggio verso reliquie. Tale aspetto ha influenzato la configurazione morfologica delle fabbriche, con il progetto di planimetrie a più navate suddivise tra corpi architettonici
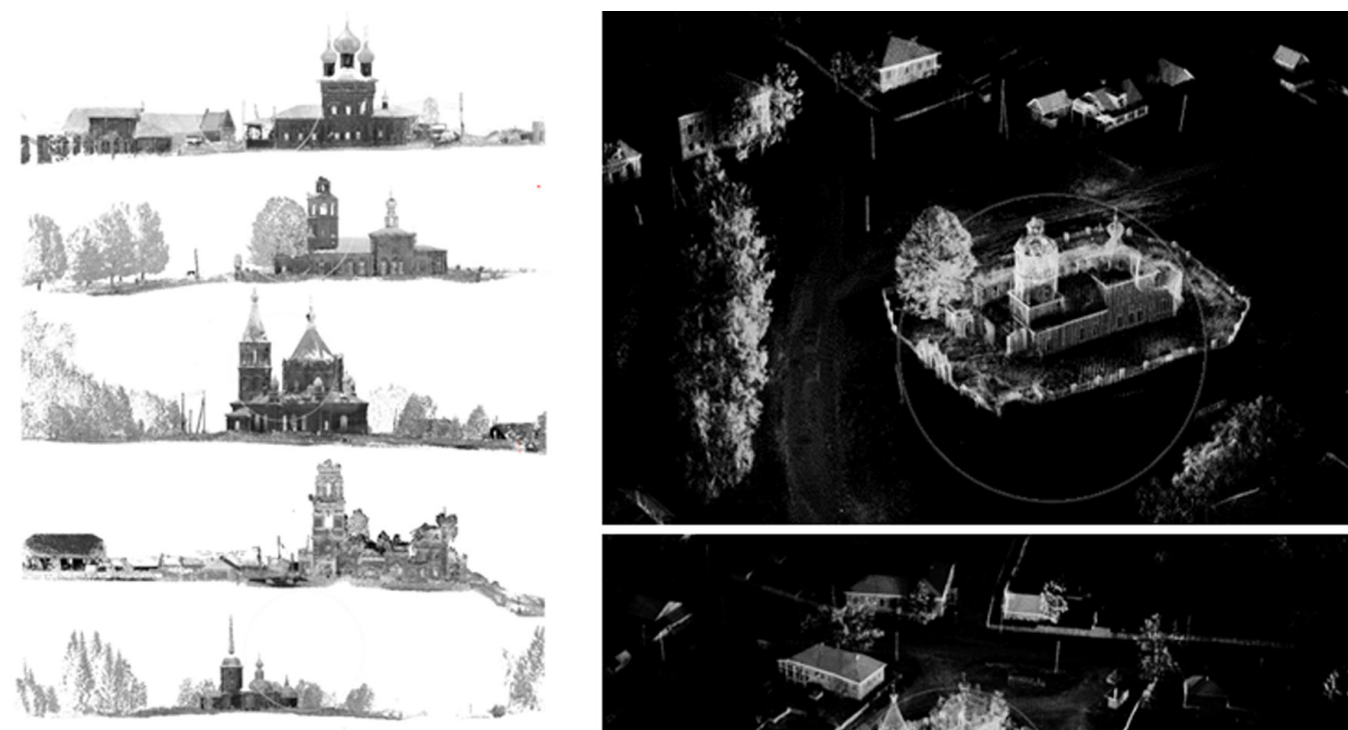

Fig. 4. Archivio digitale di dati spaziali morfometri dei siti monumental dell'Upper Kama: la molteplicità e l'estensione delle aree di rilievo dialogano con le finalità d rappresentazione dei liveli di $\mathrm{OCH}$, influenzando dimensione e caratteristiche dei singoli database.
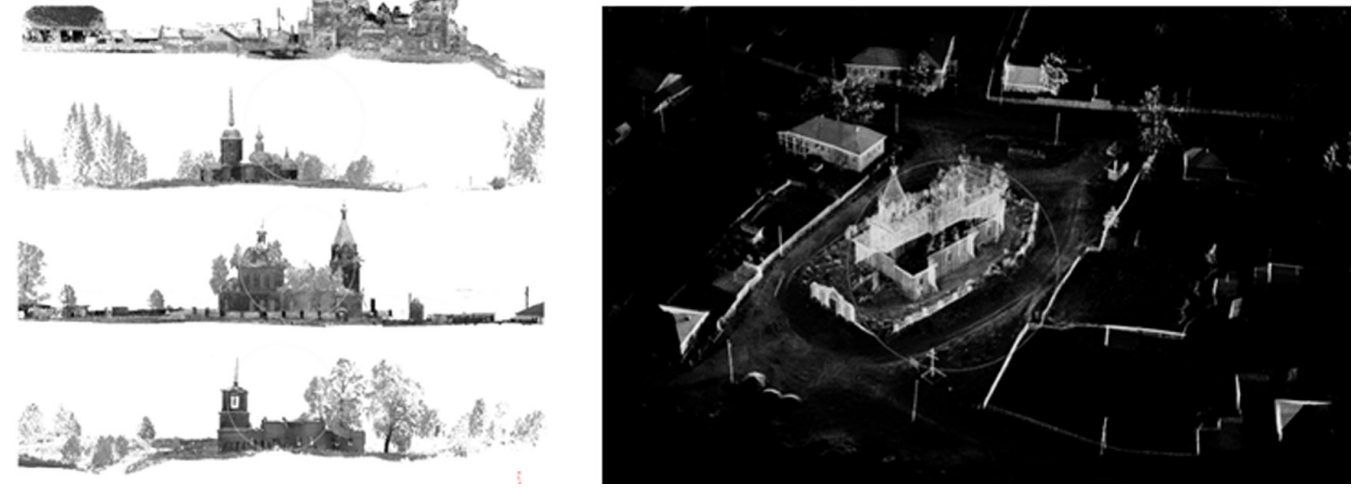
quali il nartece, con torre campanaria, il vestibolo e il corpo principale, con blocchi murari di aggregazione in corrispondenza di cappelle laterali ed espansioni. La configurazione dei complessi prevedeva anche un affiancamento del monumento principale con un'eventuale chiesa invernale, a navata unica e più contenuta per permetterne il riscaldamento, e cappelle secondarie, cripte o mausolei a completamento del recinto religioso. L'estensione dei complessi era inoltre legata al loro impatto sul paesaggio e sui flussi insediativi, spesso tale da relazionare il circostante impianto di sviluppo dei corrispondenti centri urbani. Con il periodo sovietico, la richiesta di conversione del territorio a fini infrastrutturali e di produzione energetica o alimentare [8] ha definito un decadimento del ruolo esercitato da tali siti nel bacino culturale dell'Upper Kama. II conseguente stravolgimento degli impianti costruttivi, forzatamente adeguati alle nuove esigenze, ha finito con il decretarne un prevalente abbandono, fino alle condizioni di rovina e collasso.

A fronte di tale contesto, l'azione di ricerca del progetto europeo H2020-RISE-PROMETHEUS [9] è mossa a sviluppare un protocollo di documentazione e rappresentazione per le Cultural Heritage Route partendo dall'Upper Kama come caso pilota di elaborazione di un archivio di librerie di moduli e modelli architettonici rilevati nel bacino. La digitalizzazione, archiviazione e gestione delle informazioni sui siti è avanzata attraverso una rappresentazione orientata all'unificazione dei linguaggi grafici, verso processi di ri-applicazione ed interazione dei dati fino alla gestione collaborativa delle unità costruttive per l'implementazione alla scala territoriale (fig. 4).

\section{Strumenti di rappresentazione della memoria per la pianificazione territoriale}

Nell'immagine odierna dei monumenti dell'Upper Kama i segni del tempo appaiono come una caratteristica forte e identificativa, per quanto questi comportino un lento deperimento delle strutture che convergerà inevitabilmente nella scomparsa del patrimonio.

Mentre nelle città, l'insieme caotico dei flussi ha rimescolato le tipologie architettoniche creando nuove mescolanze ed ibridazioni, nei villaggi l'immagine del luogo rimane incontaminata. In entrambi i casi rimane chiaro il fulcro identitario che trova la sua immagine nell'architettura religiosa ortodossa, la quale mantiene la sua individualità come luogo centrale di aggregazione e simbolo identificativo all'interno di nuove espansioni territoriali. Individuato nella struttura della chiesa il carattere distintivo del luogo, il progetto ha sviluppato una prima analisi per la definizione di un protocollo di rappresentazione che permettesse di descrivere e lasciare inalterata nella memoria storica l'immagine del territorio dell'Upper Kama, restituendo attraverso la rappresentazione grafica la connessione che intercorre tra territorio, città e architettura religiosa. Tali connessioni vanno a definire un patrimonio diffuso, isolato all'interno delle singole realtà territoriali. L'immagine del luogo è rappresentata a seguito di un processo di sintesi della memoria percettiva che compie il soggetto osservante l'atto che Simmel definirebbe come Stimmung [10], la rielaborazione delle immagini seriali che il soggetto registra osservando un determinato spazio fisico filtrando autonomamente

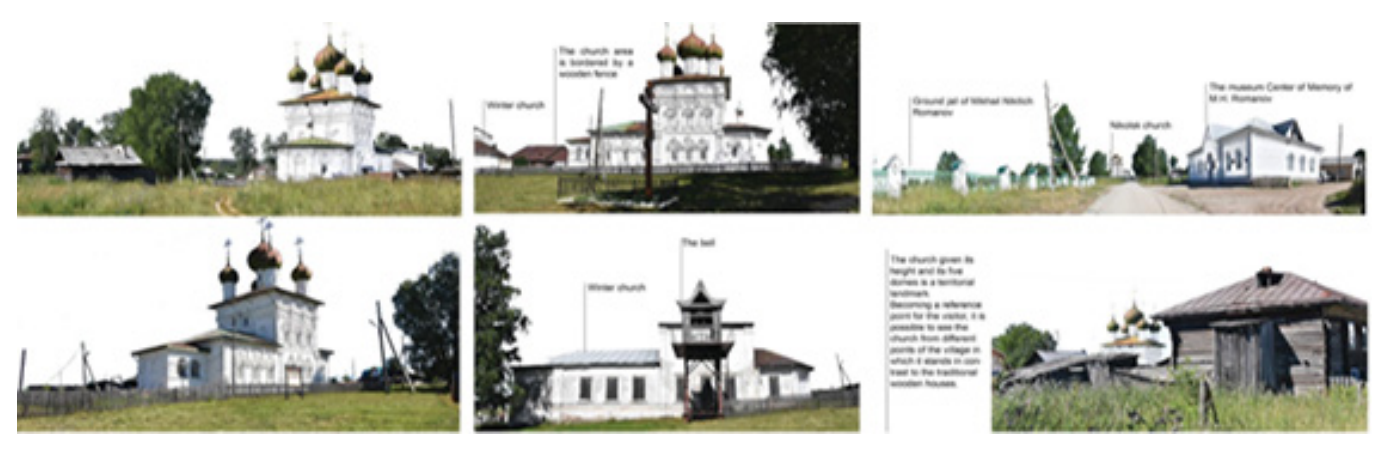


Fig. 6. Database digitale morfo-metrico da rilievo con laser scanner terrestre La pianificazione dell'acquisizione si è concentrata sull'adeguatezza centrata sull adeguatezza in in termini di dettaglio, mantenuto al livello di estensione, dedicata sia al complesso che al sito di interno.
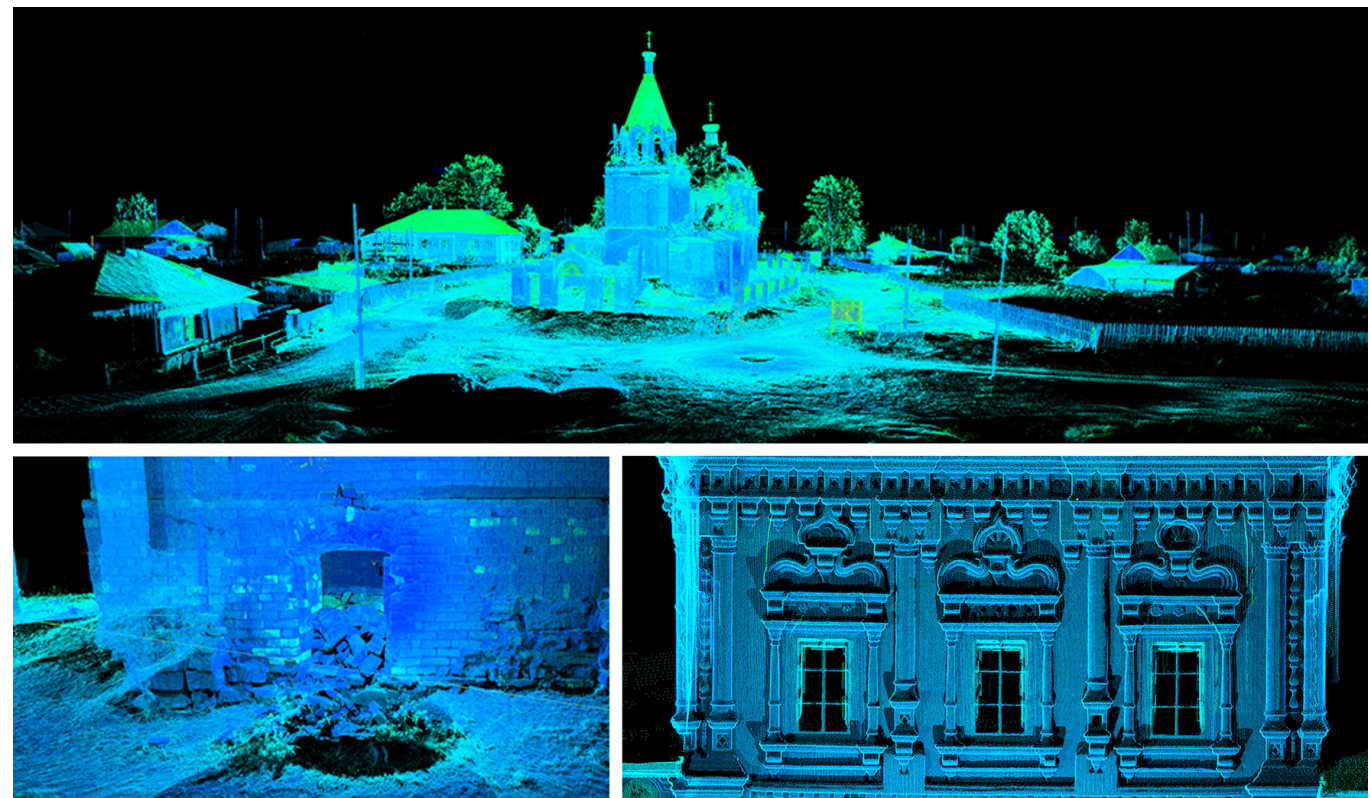

i dati, condizionato dalle proprie esperienze, disegna all'interno della sua memoria una rappresentazione di un'immagine sintetica (fig. 5).

Durante il primo anno il progetto ha risposto l'obiettivo di documentare lo stato di conservazione di tutte le strutture presenti lungo la rotta dell'Upper Kama attraverso il loro censimento. Individuati e catalogati all'interno di un abaco categorizzato gli ottanta complessi religiosi dislocati tra Cherdyn, Usolye e Solikamsk sono iniziate le operazioni di un rilievo integrato sia del singolo complesso religioso che del sistema territoriale a contorno, tramite la differenziazione delle metodologie di acquisizione.

Per il sistema territoriale sono stati utilizzati strumenti per la copertura ad ampio raggio di ripresa, un laser scanner di tipo mobile [I I] per l'identificazione e la strutturazione dei percorsi di interconnessione territoriale e urbana, oltre alla strutturazione di piani di volo con sistemi UAV [12] per la restituzione di un modello fotogrammetrico territoriale ad integrazione dei dati metrici acquisiti. Parallelamente è stata strutturata una campagna fotografica volta sia all'acquisizione del macrosistema territoriale che alla descrizione del singolo complesso religioso sino a scendere all'analisi del dettaglio del singolo elemento costruttivo

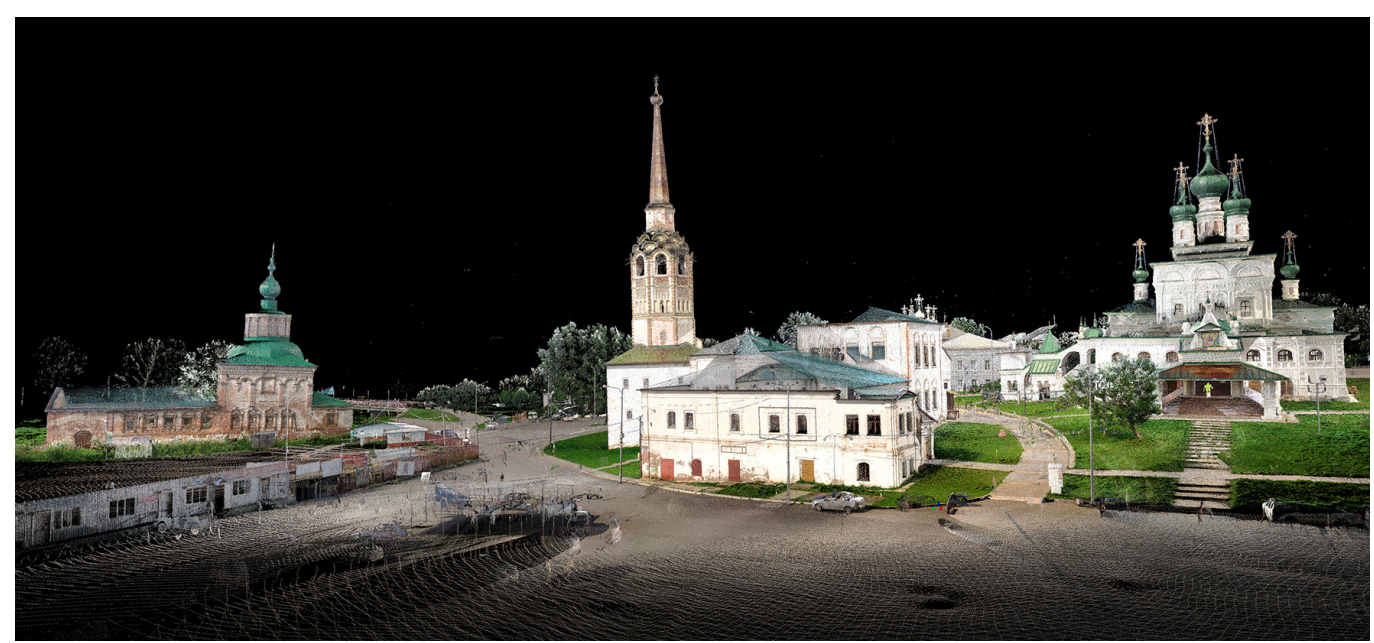

Fig. 7. Database riunificato del centro storico di Solikamsk: la referenziazion dei monumenti e delle rotte di collegamento permette una migliore comprensione delle qualità di pianificazione e servizio del sistema territoriale, ma richiama moli estese di dati di complessa gestione e manipolazione per l'analisi. 
Fig. 8. Strategie di rilevamento territoriale applicate alle Cultural Heritage Routes: rilevamento fotogrammetrico da camera aerea, applicato al sito urbano al monumento; prodotti di nuvole di punti RGB, meno dettagliate ma più estese nell'archiviazione dei dati spaziali e morfo-

Fig. 9. Strategie di rilevamento territoriale applicate alle Cultural Heritage Routes: rilevamento mobile tramite LiDAR KAARTA: il concetto d traiettoria di acquisizione e di autoreferenziazione nello spazio supporta la documentazione di sistemi estesi concentrando il rilievo sulla rotta di connessione oltre che su monumento, contribuendo anche alla speditività delle operazioni sul campo.

Fig. 10. Individuazione degli apparati di scomposizione del monumento e della rotta all'interno delle schede di catalogazione censuaria, adatte alla compilazione in sito tramite app IOS. e decorativo architettonico, al fine di creare una libreria di dati per la definizione e il censimento degli elementi caratterizzanti l'edificio. II dato territoriale è stato integrato attraverso la strutturazione di campagne di rilievo laser scanner (FARO serie SI 50) per la restituzione del dettaglio architettonico. (figg. 6-9)

L'ottimizzazione e la raccolta dati per la strutturazione di librerie digitali è seguita dall'implementazione informativa con l'annessione delle conoscenze sulle tecnologie di costruzione e sugli archivi censuari relativi all'architettura, insieme alle pratiche di pianificazione storica e territoriale raccolti all'interno di schede tecniche compilative utilizzate come base per la definizione dei criteri descrittivi per la strutturazione di un linguaggio di modellazione digitale univoco (fig. I0).
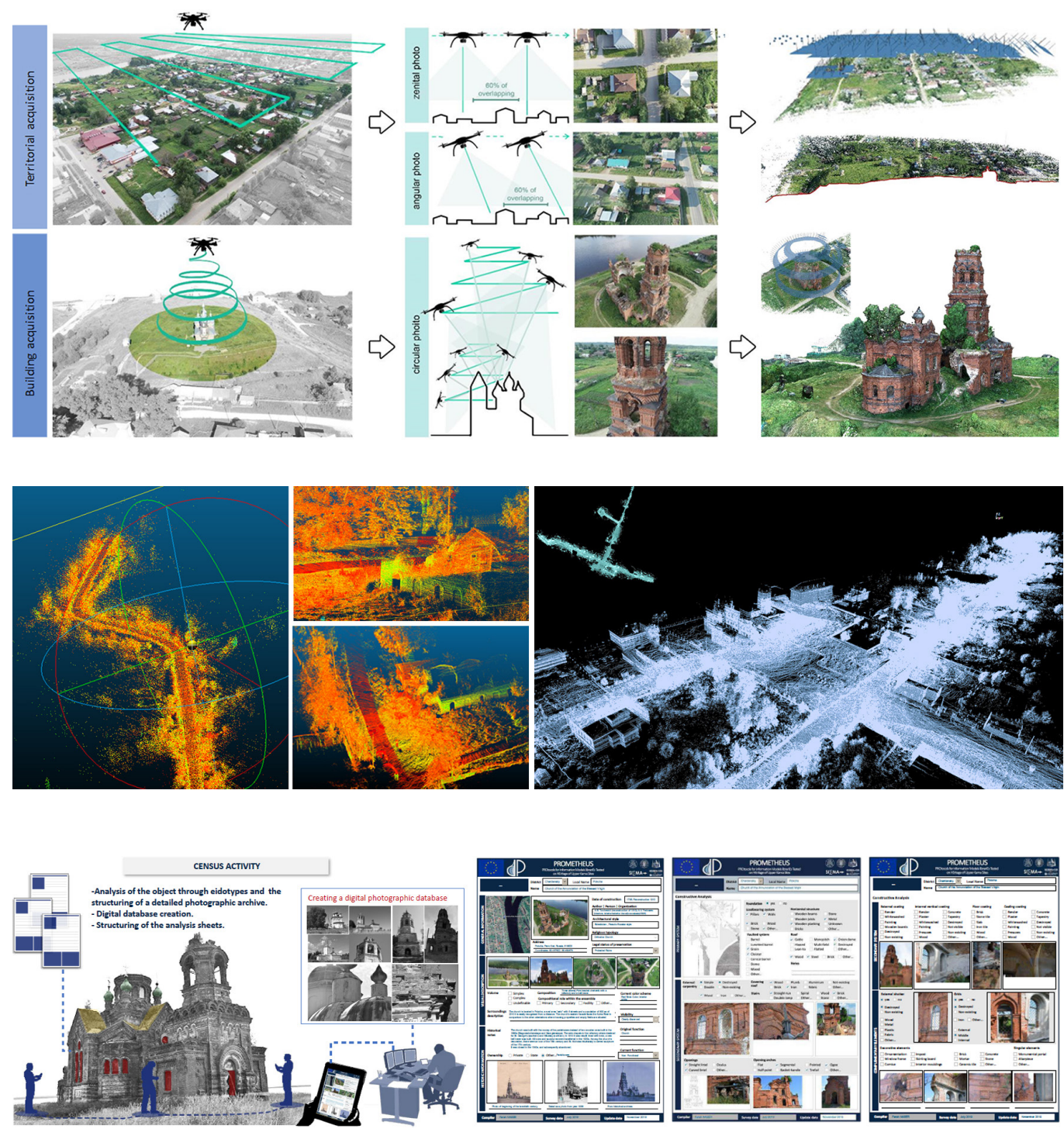

\section{Modelli informativi e paesaggi territoriali}

II tema della riproducibilità del patrimonio storico dislocato all'interno di un vasto sistema territoriale tiene il dibattito aperto sulle problematiche di strutturazione e condivisione di una tipologia di linguaggio unificato fatto di segni e simboli che possa tradurre il sistema di interconnessione territoriale attraverso la sua codificazione in uno schema formale generalizzabile. 
Attraverso la disciplina della rappresentazione, viene definito un linguaggio grafico nel quale la complessità del reale è ridotta in favore di una comunicazione selettiva delle informazioni. II disegno esplicita quelle esigenze descrittive che animano la forma della comunicazione e quindi operano attraverso la sintesi grafica la trasmissione dell'informazione.

A tal fine risulta necessario un processo di analisi ed identificazione dell'antologia semantica degli edifici censiti che sono parte del patrimonio diffuso dell'Upper Kama, per strutturare una gerarchia per i modelli geometrici sulla quale impostare la gestione delle informazioni raccolte durante le fasi di acquisizione ed indagine d'archivio (fig. I I).

Nell'obiettivo di strutturare un piattaforma di raccolta di informazioni accessibile e interoperabile, tale tipo di approccio metodologico del Building Information Modeling applicato al Cultural Heritage apre un potenziale ventaglio di applicazioni, permettendo di confluire la quantità di dati e la possibilità di gestione per l'integrazione a scale superiori a quella del singolo edificio, estendendosi dall'ambito dell'analisi architettonica a quello della progettazione e programmazione urbana, territoriale, e soprattutto infrastrutturale, contesto in cui tale strategia può rappresentare un cambio di paradigma finalizzato a gestire in maniera più efficace la complessità della struttura territoriale.

Il protocollo, in fase di progettazione, specifica le linee guida a supporto della modellazione, attraverso la strutturazione di un abaco condiviso diversificato sulla base della definizione di differenti LOD di rappresentazione per il dettaglio del modello per differenziare i livelli di indagine, codificando ogni elemento in specifiche categorie di modello.

Fig. I I. La scomposizione semantica degli elementi per lo studio delle categorie degli elementi ricostruiti.

Fig. 12. Complessità del parametri e dei collegamenti tra il progetto di sistema informativo e le complessità architettoniche. Prime considerazioni sulla costruzione di supporti di modellazione geometricamente semplificati, pur se di alto contenuto informativo, che possano permetter la gestione dei modell descrittivi alla scala de
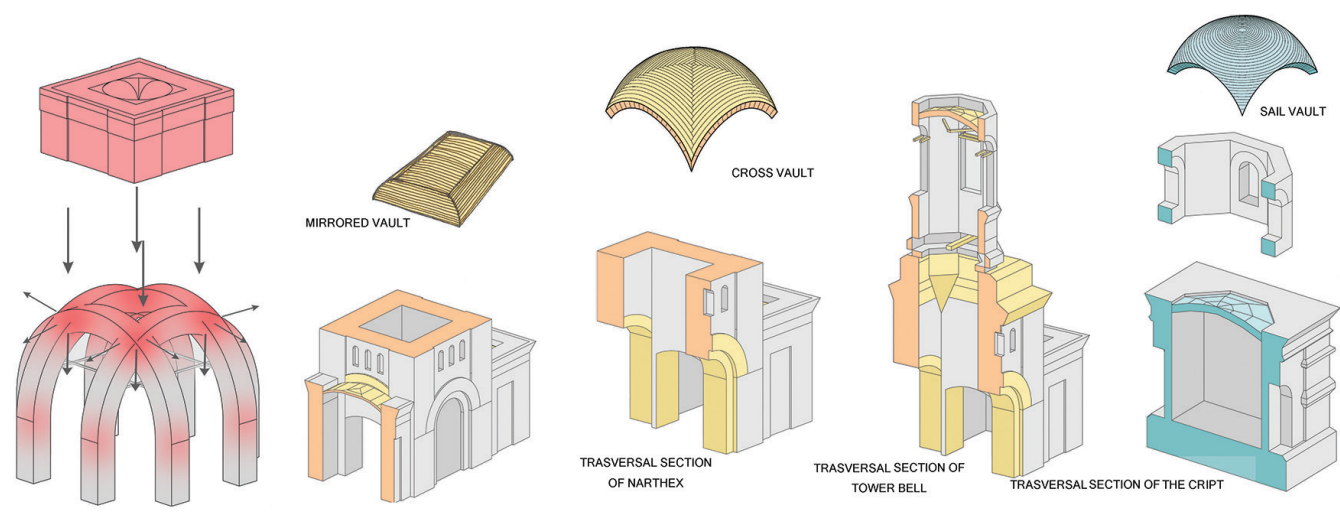

OF NARTHEX TOWER BELL
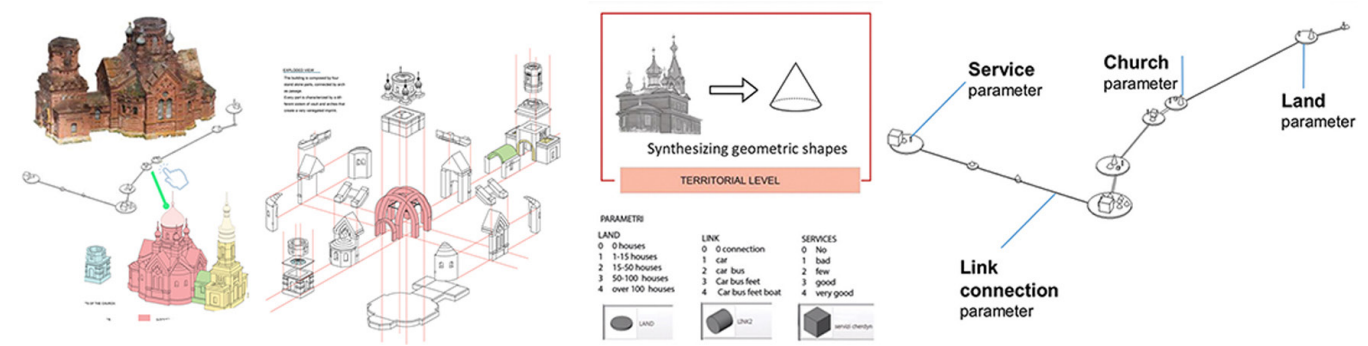

Una differenziazione nel linguaggio grafico del modello risulta necessaria per la predisposizione di differenti gerarchie di contenuti definite tramite l'utilizzo di forme essenziali, per la strutturazione di un sistema di modello che possa essere descrittivo della condizione territoriale.

La definizione di classi di modello che trovano, attraverso la strutturazione di abachi tipologici di famiglie, permette di poter identificare e raccogliere all'interno dello spazio tridimensionale i sistemi di interconnessione tra i complessi architettonici ed il sistema paesaggio. II modello modifica la sua forma secondo le finalità e le tipologie di descrittore che assume nel sistema globale. Quando è descrittore del sistema territoriale sarà rappresentato attraverso l'associazione di una volumetria di sintesi della forma geometrica, al contrario quando 
è descrittore del complesso architettonico, la geometria, prima sintetizzata, sarà implementata con i contenuti informativi per una lettura approfondita a disposizione di differenti figure d'intervento per la gestione architettonica e territoriale (fig. I2).

La scelta metodologica è fatta con la consapevolezza dell'ampio catalogo storico-architettonico di elementi che caratterizzano le architetture dei siti dell'Upper Kama. Pertanto, le operazioni di indagine e la scrittura di un manuale specifico di raccolta delle componenti di modello possono essere utili per la strutturazione dei processi di integrazione dei dati, tramite il confronto diretto tra oggetti reali e modelli virtuali.

\section{Note}

[I] La responsabilità redazionale del contributo è riconosciuta a Raffaella De Marco per i paragrafi I e 2, a Anna Dell'Amico per i paragrafi 3 e 4.

[2] L'idea di 'diffusività' del patrimonio culturale è oggi richiamata in merito alle crescenti esigente di sua 'accessibilità', ma deve essere intesa non solo in termini propriamente fisici del sito specifico ma più in generale nella mappatura e controllo di dati dispersi in un quadro unificato di conoscenza, monitoraggio e pianificazione culturale. A tal riguardo, l'uso di archivi di informazioni e contenitori di referenziazione è crescentemente richiesto, espandendo l'influenza delle metodologie visuali di rappresentazione e aggiornandone i prodotti grafici in termini di digitalizzazione e network di metadata [Parrinello, Dell'Amico 2019, pp. 1032-1044].

[3] "Pensare oggi al tema dei Paesaggi culturali [... ] rende necessari sguardi locali e visioni globali del mondo contemporaneo". Dal momento che nel processo di globalizzazione economica e culturale, il riconoscimento dell'identità (e della alterità) di un bacino culturale si presenta come un problema cruciale, la questione della rappresentazione dei paesaggi culturali si lega a quella dello spazio ed ai suoi processi di simbolizzazione. "In ogni paesaggio coesistono dunque l'oggetto, la sua estensione fisica, il suo spazio e le rappresentazioni mentali di una comunità, nella loro reciproca articolazione problematica." II paesaggio culturale quindi "non è solo pura estensione fisica da fruire in senso tecnico/economico ma luogo in cui avviene una "mediazione culturale" che conferisce forma allo spazio." Ne consegue così "l'importanza decisiva della sua rappresentazione, non solo quella mentale anche delle sue forme concrete", sempre più incoraggiate ad una connessione intertestuale capace di riconoscere dimensioni tecnico-pratiche di tangibilità rappresentativa ed estensione geografica per l'identificazione degli apparati corrispondenti sul territorio [Salerno, Casonato 2008, pp. 12-16].

[4] II riconoscimento deriva dal report dell'incontro di esperti del World Heritage Committee WHC-94/CONF.003/INF. I3, "Routes as a part of our Cultural Heritage" (Madrid, Spagna, Novembre 1994), successivamente implementato dall'ICOMOS nella "Charter on Cultural Routes", 2008. Ë centrale il riconoscimento del patrimonio delle rotte come "sum of elements referring to a whole [...] within a joint system which enhances their significance" portando a una "dynamic conformation of cultural landscape". La recente inclusione di gran parte di queste rotte (a titolo di esempio, l'Hansa I99I, El Legado andalusi 1997, il patrimonio industriale della Valle del Reno 2002, la Rotta dei Pirenei 2003, i Siti cluniacensi 2005, la Transromanica 2007, le Abbazie cistercensi 20 I0, le Città termali storiche 20 I0, le Fortezze di Carlo V 20 I5, le città fortificate della Grande Région 20 I6) i loro programmi di documentazione circa le condizioni storiche e fisiche, il valore architettonico ed i programmi operativi di intervento per I gestione territoriale risultano ancora scarsi. I progetti esistenti si concentrano principalmente su un approccio turistico, educativo e formativo (progetti HECTOR e CERTESS, dell'Istituto europeo di percorsi culturali), mancando di un protocollo dedicato per la gestione territoriale unificata dei siti.

[5] Le ricerche di A.S. Teryohin, G. D. Kantorovich, I. V. Makovetskiy e V. A. Tsypushtanov hanno fornito un fondamentale contributo alla conoscenza del patrimonio dell'Upper Kama nella loro opera del 1970. Nel 1988 la monografia di Kostochkin ha integrato le precedenti ricerche, oltre all'archivio del Perm Scientific Restoration Atelier che dal 1986 fornisce dati affidabili sui monumenti esistenti per lo sviluppo di progetti di conservazione e restauro. Lo studio più recente sull'architettura dell'Upper Kama è condotto da W. Brumfield nella forma di report fotografico del 2012.

[6] La documentazione del patrimonio costruito dell'Upper Kama è parte di un programma di attività sviluppato dal 20 I 3 dal Professor Sandro Parrinello dell'Università degli Studi di Pavia e dalla prof. Svetlana V. Maximova della Perm National Research Polytechnic University. Tre summer schools sono state organizzate nel 2015, 2016 e 2018 coinvolgendo professori, ricercatori e studenti di università internazionali, e supportate dal contributo del Russian Academy of Painting, Sculpture and Architecture, Stroganov Chambers Historic Architectural Museum di Usolye e dall'amministrazione di Usolye.

[7] II Perm Krai Center for Monuments Protection si occupa oggi del patrimonio dell'Upper Kama per la sua preservazione e lo sviluppo di progetti di intervento e protezione, come il caso della Protecting Zone per il centro storico di Usolye.

[8] A seguito dell'ascesa sovietica, numerosi complessi furono abbandonati o videro una riconversione dei loro spazi per altre funzioni, prevalentemente di produzione energetica (come la Church of the Annunciation of the Blessed Virgin di Pokcha convertita in centrale elettrica nel 1940, il caso della Church of the Exaltation of the Holy Cross a Solikamsk, abbandonata nel 1929 e successivamente trasformata in un birrificio, e la Church of the Epiphany a Cherdyn, oggi sede di un panificio). L'evento centrale è rappresentato dalla costruzione della stazione elettrica sulla Kama nel 1954, con l'esondazione del fiume e la variazione del rapporto idrogeologico territoriale tra i livelli di falda ed i siti di fondazione dei complessi, causa di evidenti dissesti e condizioni di instabilità che ne contraddistinguono oggi la condizione di patrimonio in emergenza.

[9] II progetto PROMETHEUS raccoglie e sviluppa tali ricerche all'interno del programma di finanziamento europeo Horizon 2020-R\&I-RISE- Research \& Innovation Staff Exchange Marie Skłodowska-Curie. II progetto (coordinato dal prof. S. Parrinello dell'Università di Pavia) prevede la collaborazione tra tre università internazionali (Università degli Studi di Pavia, Universitat Politecnica de Valencia, Perm National Research Polytechnic University) e due imprese (SISMA srl, EBIME sl.). II progetto include la partecipazione di I I Experienced Researchers e 5 Early-Stage Researchers, oltre a studenti ordinari e di master in ingegneria e architettura delle università coinvolte. This project has received funding from the European Union's Horizon 2020 research and innovation programme under the Marie Skłodowska-Curie grant agreement No 821870.

[ I0] “Il paesaggio, diciamo, si costituisce quando i vari elementi naturali distesi uno vicino all'altro sul suolo terrestre si riuniscono in una unità di genere molto particolare, differente rispetto a quella intravista dallo scienziato con il suo pensiero causale, 
dall'adoratore della natura con il suo sentimento religioso, dal contadino o stratega con il loro approccio teologico." Simmel definisce la Stimmung, una qualità del paesaggio come sentimento proprio umano. Senza tale sentimento il paesaggio sarebbe solo una somma di elementi naturali. Questo però non lo è dal momento che l'uomo contemplandolo ha un atteggiamento di elaborazione, nel senso che percepisce gli elementi della natura e li unifica. Facendoli propri, assorbe la sostanza data dalla natura e la rielabora attraverso l'atto della contemplazione [Simmel 1985]

[I I] II laser scanner mobile KAARTA Stencil basa il suo sistema di acquisizione dato su una rielaborazione dei dati LIDAR e IMU per la localizzazione. II sistema utilizza Velodyne (VLP-16) collegato a una IMU MEMS a basso costo e un computer di elaborazione per la mappatura e la localizzazione in tempo reale. La strumentazione KAARTA e FARO è messa a disposizione del laboratorio PLAY del Dipartimento di Ingegneria Civile e Architettura dell'Università di Pavia.

[I2] Per l'acquisizione territoriale per l'elaborazione aereo fotogrammetrica è stato utilizzato un drone serie DJI Phantom 4 Pro, messo a disposizione dal laboratorio DAda Lab.

\section{Riferimenti bibliografici}

Brumfield William Craft (1993). A history of Russian architecture. Cambridge: University press, pp. 664.

Brumfield William Craft (2008). The architectural heritage of Solikamsk and the Northern Districts of Perm Province. In Cahiers slaves. Routes et Chemins slaves, sous la direction de Laure Troubetzkoy et Fracis Conte, 10, pp. 317-355.

Di Giulio Roberto et al. (2017). Integrated data capturing requirements for 3D semantic modelling of cultural heritage: the inception protocol. In ISPRS - International Archives of the Photogrammetry, Remote Sensing and Spatial Information Sciences. XLII2NW3. 25 I-257. 10.5 I94/isprs-archives-XLII-2-W3-25I-2017.

Jackob Michael (2019). II paesaggio. Bologna: II Mulino.

Jordan Isabel et al. (20।8). Protocol to Manage Heritage-Building Interventions Using Heritage Building Information Modelling (HBIM). Sustainability. I0.908. 10.3390/sul 0040908.

Kioussi Anastasia et al. (2012). Integrated documentation protocols enabling decision making in cultural heritage protection. In Journal of Cultural Heritage, 14 (3), pp. $14 \mid-146$.

Kostochkin V.V., (1988). Cherdyn, Solikamsk, Usolye. Moscow: Stroiizdat.

Parrinello Sandro et al. (2019). Documenting the Cultural Heritage Routes. The creation of informative models of historical Russian churches on Upper Kama Region. In Int. Arch. Photogramm. Remote Sens. Spatial Inf. Sci., XLII-2M I 5, 20 I 9, pp. 887-894.

Parrinello Sandro, De Marco Raffaella (2019. Integration and modelling of 3D data as strategy for the structural diagnosis in Endangered Sites. The study case of Church of the Annunciation in Pokcha (Russia). In Proceedings of 2019 IMEKO TC-4 International Conference on Metrology for Archaeology and Cultural Heritage. Firenze: IMEKO, pp. 223-228.

Parrinello Sandro, Dell'Amico Anna (2019). Experience of Documentation for the Accessibility of Widespread Cultural Heritage. In Heritage, 2(I), pp. I032- 1044.

Parrinello Sandro, Cioli Federico (2020). Establishment of a complex database for the study of Cultural Heritage through the reading and analysis of the traditional architecture of Upper Kama. In Kremers Horst. In Digital Cultural Heritage. Cham: Springer, pp. 5|-6|.

Parrinello Sandro, Cioli Federico (2018). Un progetto di recupero per il complesso monumentale di Usolye nella regione della Kama Superiore. In Restauro Archeologico, I, 2018, pp. 92-III.

Parrinello Sandro, Maksimova Svetlana, Mezenina Kseniia (2015). Historic environment architectural survey with the use of digital technology. Vestnik Permskogo Nacional'nogo Issledovatel'skogo Politehničeskogo Universiteta. In Urbanistika, I, pp. $102-117$.

Parrinello Sandro, Maksimova Svetlana V. (2016). Contemporary methods of urban environment architectural survey. Perm: Polytechnic University.

Parrinello Sandro, Picchio Francesca (2014). Recomposing the landscape using structure from motion survey in the village of Korza, Karelia (Russia). In SCIRES, 4, 2, pp. $29-42$.

Simmel George (1985). Philosophie der Landschaft, in "Die Güldenkammer", I 9 I 3. (Trad. it. Filosofia del paesaggio, in Id., II volto e il ritratto. Saggi sull'arte, Bologna: il Mulino.

Teryohin A.S. et al. (1970). Monuments of stone architecture of XVI-XVIII in Solikamsk. Perm.

\section{Autori}

Raffaella De Marco, Università di Pavia, raffaella.demarco@unipv.it

Anna Dell'Amico, Università di Pavia, anna.dellamico@unipv.it

Per citare questo capitolo: De Marco Raffaella, Dell'Amico anna (2020). Connettere il territorio tra patrimonio e informazione: banche dati e modelli per le Cultural Heritage Routes/Connecting the territory between Heritage and Information: databases and models for the Cultural Heritage Routes. In Arena A., Arena M., Brandolino R.G., Colistra D., Ginex G., Mediati D., Nucifora S., Raffa P. (a cura di). Connettere. Un disegno per annodare e tessere. Atti del $42^{\circ}$ Convegno Internazionale dei Docenti delle Discipline della Rappresentazione/Connecting. Drawing for weaving relationships. Proceedings of the 42th International Conference of Representation Disciplines Teachers. Milano: FrancoAngeli, pp. $2058-2077$. 


\title{
Connecting the Territory between Heritage and Information: Databases and Models for the Cultural Heritage Routes
}

\author{
Raffaella De Marco \\ Anna Dell'Amico
}

Abstract

The contribution deals with the 'connection' between built heritage and territory, deepening the forms and roles of multidimensional "representation" declined between 'architecture' and 'cultural route'. Cultural Heritage Routes [ICOMOS, 2008] assume a key role as collectors of a heritage that goes beyond its geographical and political boundaries. Through the experiences offered by H2020-RISE-PROMETHEUS project (2019-202I), a reflection is conducted on the widespread heritage of the Upper Kama basin, in Russia, as a pilot case on the cultural route of religious architecture and settlements of the salt trade between Europe and Asia.

The project is aimed at developing a programmatic Charta for Territorial Information Models replicable in European contexts. The aim is to highlight the multiple levels of knowledge stratified on the single monument and site, from the construction system to the architectural complex till to reach the territorial network of relations with the cultural basin, in a process of morphological, technological and typological semantisation that allows to coordinate its combined management of memory, information and intervention on the object.

Keywords

widespread heritage, digital survey, information systems, Cultural Heritage Routes, Upper Kama.

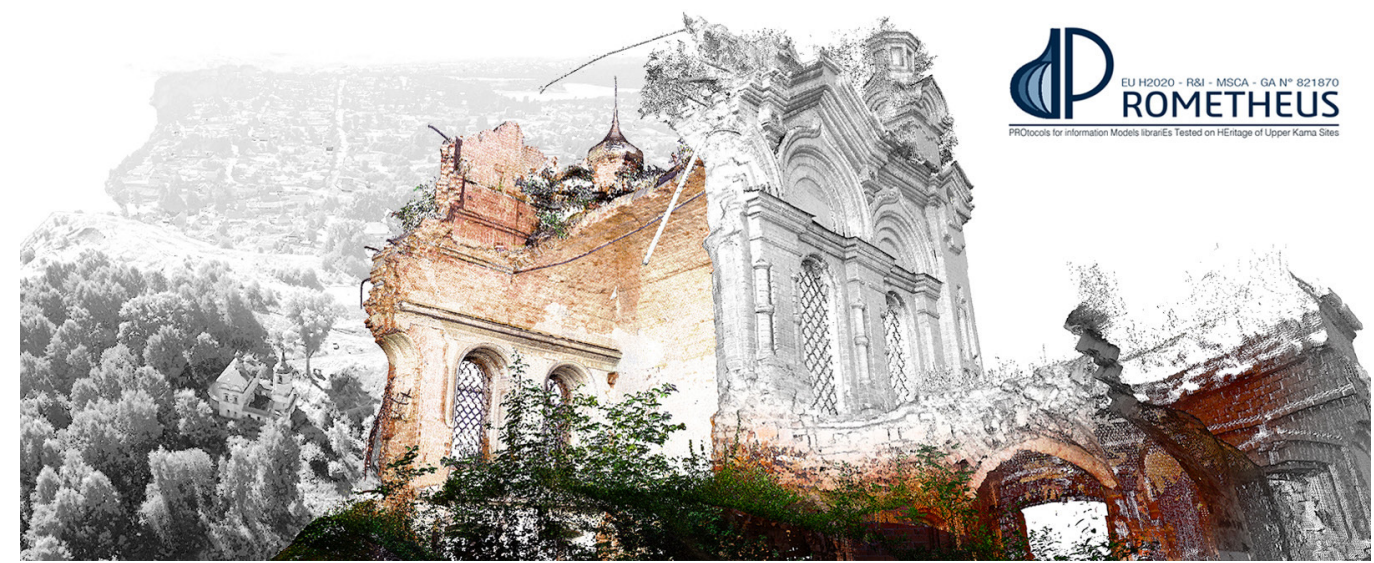




\section{Cultural Heritage Routes as a connection scenario between territory and monument}

The study of the connections between built heritage and territory is based on the analysis of the cultural imprint of mutual influence between the site and the landscape network where it belongs $[\mathrm{I}]$.

The advised characters are at the same time tangible, materially represented by the constructive and infrastructural signs, and intangible, in the memory of traces and socio-economic networks that no longer exist but still influence the location of monuments and routes. Between settlements and stylistic flows based on 'basin' connections, a classification of images and information on a type of "widespread heritage" [2] is recognized with increasing emphasis, generated by the intertwining of geographical and cultural crossroads, where a deeper relationship between sites and monuments belonging to the scale of the territory still emerges from the form of anthropic intervention on the landscape. The recognition of extensive networks of contamination between cosmopolitan traditions and local territories, in particular between Europe and closer continents, classifies scenarios capable of affirming and maintaining specific characteristics of identity. The understanding and structuring of these characteristics are necessary to operate their management and enhancement, conforming the local heritage between memory and planning as a specific 'cultural landscape' [3]. The recognition of 'cultural routes' [4], actually at the centre of communitarian programs for their enhancement and management, has sensitized the shared European heritage on the spatial and temporal dynamism of its architectural 'basins', highlighting the historical phenomena of exchange and dialogue between peoples and regions with the contemporary frameworks of territorial planning and management. Continuity through space and time thus becomes the key to classify Cultural Heritage along Cultural Heritage Routes, defined as an inclusive category between material heritage and territorial memory capable of understanding tangible but dispersed values of settlement processes. The connective qualities testified by the visual and constructive characteristics of architecture and place are researched and classified as parametric values of synthesis and understanding of territorial culture, dynamically conforming cultural landscapes over time (fig. I).

Fig. I. Preliminary classification of the Objects of Cultural Heritage (OCH) for the territorial management of the Upper Kama Cultural Heritage Route: Monument, Ensemble and Site define levels of interaction and development of architectural, landscape descriptors and infrastructures for the analysis of the monumental nucleus of Upper Kama.

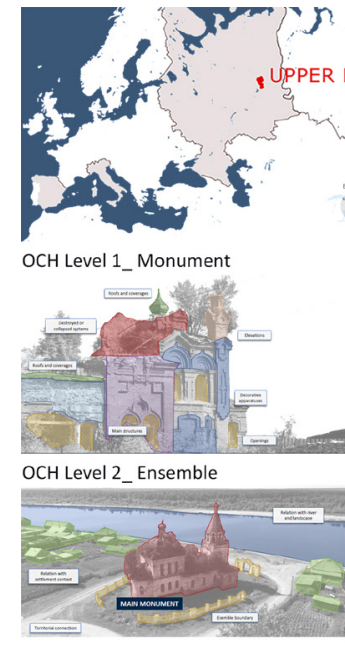

OCH Level 3_Site
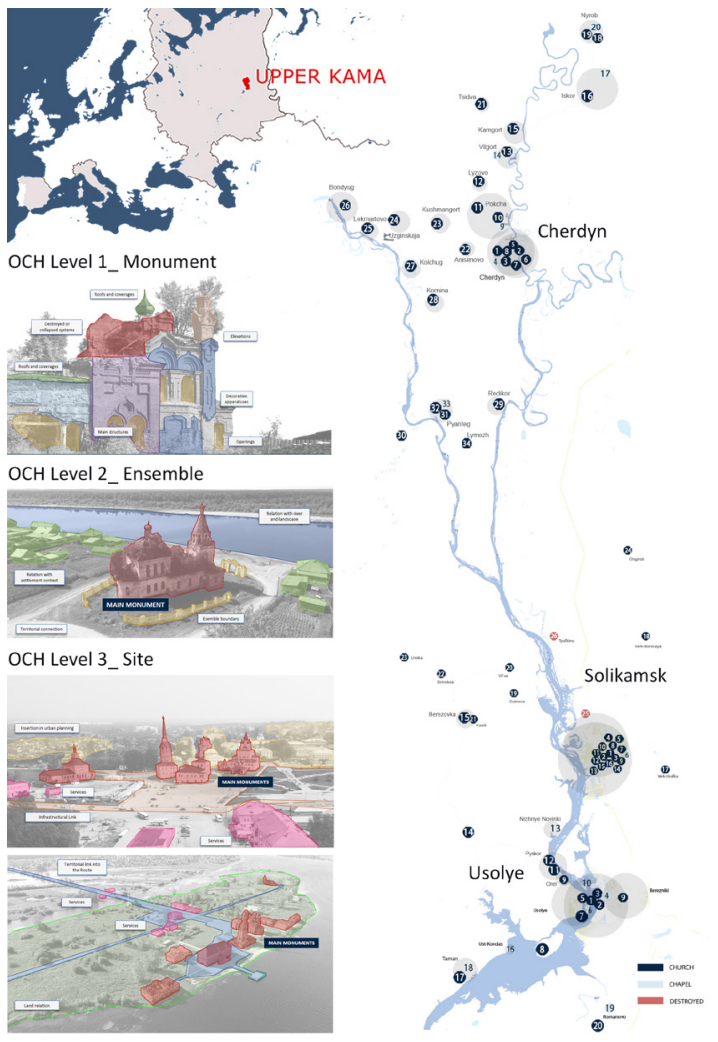
Fig. 2. Monumental sites of Upper Kama and relationship with the landscape: from above on the left, Church of Saints Peter and Paul in Taman (1912), Church of St. Nicholas in Nyrob (1704), Chapel of Kazan Mother of God in Pokch (1913), Church of Nativity in Iskor (1793), Church of St. Nicholas in Uzhginskay (1874), Church of Annunciation of the Blessed Virgin in Pokcha (1785). Church of Presentation of Blessed Virgin in Kamoort (1915) Church of Church of the Exaltation of the Holy Cross in Bondjug (1858), Church All Saints in Cherdyn (1817).
Thus, the representation is entrusted with the recognition of characteristics and values of connection between physically distant but culturally united sites, supported by spatial orders, hierarchies and geometries of forms connected themselves in a direct derivation from constructive technologies, materials and styles as tangible elements of a physical nature of the sites and a typological identification of the route. Therefore, the geographical boundaries help to visualize the Cultural Heritage Route, determining its path or development over time, but cannot contain its cultural phenomenon, assimilated by the method of knowledge of its characters and expressible only with a process of synthesis of its typological contexts, such as that operated through the drawing.
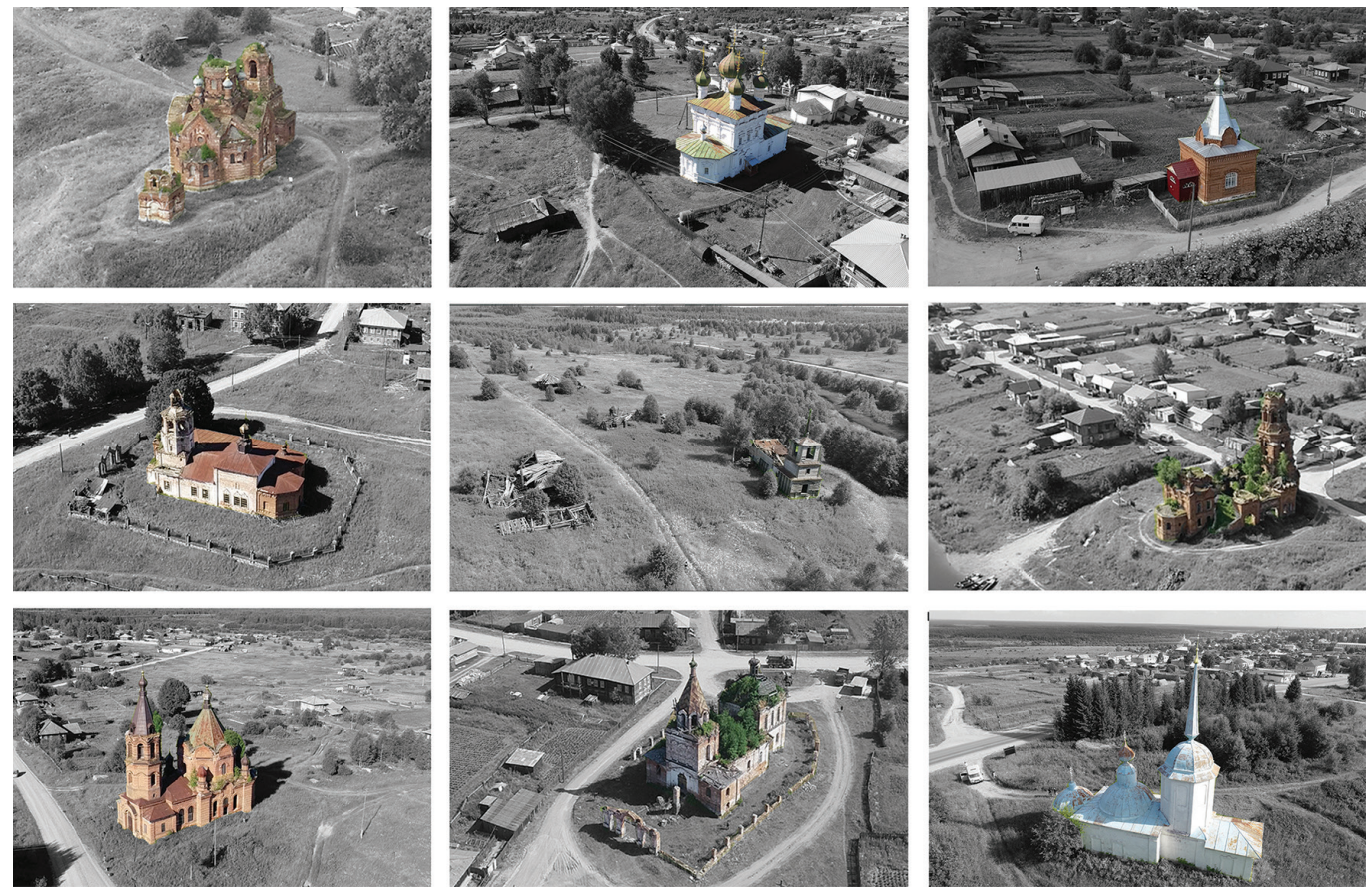

Analysis of the architectural characters for the representation of cultural routes: the case of Upper Kama, Russia

The pilot case of Upper Kama, already interested by landscape studies of the last decades [5] and from many years of international research experience on the digital documentation of some historical complexes [6], has been identified as an emblematic Cultural Heritage Route of coexistence between monumental architecture and widespread territorial criticalities (fig. 2), reflecting the stylistic and formal imprint of European culture beyond its political borders. In these terms, its sites collect a catalogue of architectural characters, stylistic varieties, construction solutions and a multiplicity of building materials, with related pathologies of degradation and conservation, such as to characterize the identity of its basin and the corresponding cultural landscape (fig. 3).

The Upper Kama region, developed around the Kama River basin, extends north of Perm Krai for about 7,000 square kilometres, west of the Ural Mountains in European Russia. Mineral resources and the role of commercial crossroads of the imperial period led Upper Kama to an intense cultural and architectural development between the I5th century, with the first industrial settlements, and the I 8th century, till the rapid decline with the Russian Revolution of October (1917). The discovery of local salt mines by merchant families, such as the Stroganovs or the Golitsyns, has decreed the development of urban settlements cha- 
Fig. 3. The identification and classification of stylistic features highlights a

mixture of local, tartar and European shapes and gemetries, also developed in traditional zuchcovii and sekirovidnymi decorative motifs, as well as a com bination of prevaling wowith related pathologies of degradation.
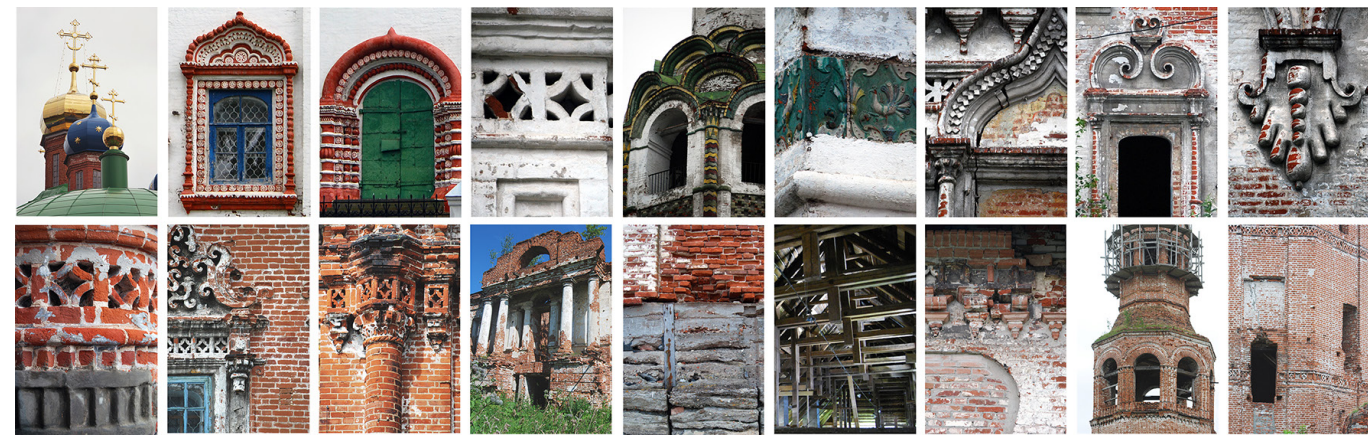

racterized by monumental Orthodox complexes, churches, bell towers and family chapels, attracting continental architects and artists to define a new and dominant architectural profile in the river landscape. The salt market, which from Europe reached the Urals to trade with China, led to the spread of numerous urban centres and to the dispersion of their monumental sites, associated with the districts of Solikamsk (I430), Cherdyn (I535) and Usolye ( 1606 ) and still today assigned to a fragmented territorial administration directed to the central control of Perm Krai [7].

The settlement imprint, more stabilised by the commercial network than the villages of the countryside, has led to a cultural phenomenon of transformation from the typical wooden architecture to brick and stone masonry solutions of construction, together with unitary stylistic themes of form and decoration derived from the Muscovite Baroque and the European influences. Upper Kama sites are characterized by monumental and often isolated religious architectures, originally belonging to larger ceremonial complexes or dedicated to

the pilgrimage for relics. This aspect has influenced the morphological configuration of the
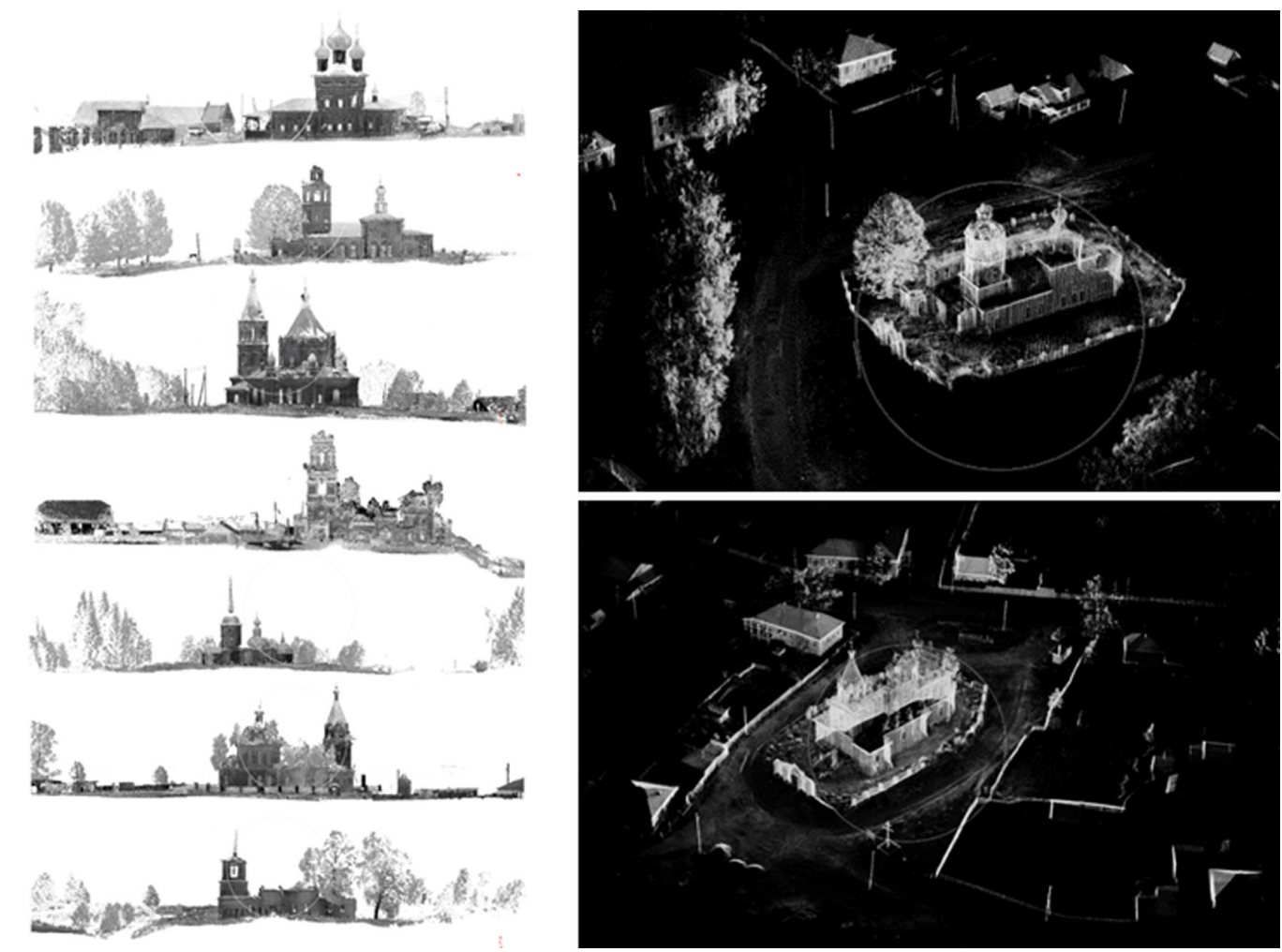

Fig. 4. Digital archive of morphometric spatial data of the monumenta sites in Upper Kama: the multiplicity and extension of the relevant areas dialogues with the purposes of representation of $\mathrm{OCH}$ levels, influencing the size and characteristics of 
built sites, with the design of plans with multiple naves divided between architectural blocks such as the narthex, with the bell tower, the vestibule and the main body, also including secondary blocks of aggregation in correspondence of side chapels and expansions. The configuration of the complexes also included a possible winter church annexed to the main monument, with a single and more contained nave to allow the function of heating, and secondary chapels, crypts or mausoleums to complete the religious ensemble. The extension of the complexes was also linked to their impact on the landscape and on the settlement flows, often so deep to relate the surrounding development system of the corresponding urban centres. With the Soviet period, the request of territorial conversion for infrastructure and energy purposes or food production [8] has defined a decline in the role played by these sites in the Upper Kama cultural basin. The consequent change of the construction systems, forced to adapt to the new requirements, has caused a prevalent abandonment of the buildings, till to reach the conditions of ruins and to collapse.

In this background, the research action of the European project H2020-RISE-PROMETHEUS [9] moves to develop a documentation and representation protocol for the Cultural Heritage Routes starting from Upper Kama as a pilot case, for the processing of an archive of libraries of architectural modules and models found in the basin. The digitization, archive and management of information on the sites is advanced through a representation oriented towards the unification of graphic languages, enhancing processes of re-application and interaction of data to the collaborative management of the construction units for the implementation at the territorial scale (fig. 4).

\section{Instruments of representation of the memory for the territorial planning}

In the present image of Upper Kama monuments, the signs of time appear as a strong and identifying feature, although they entail a slow deterioration of the structures that will inevitably converge in the disappearance of this heritage. While in the cities, the chaotic set of flows has remixed the architectural typologies creating new mixtures and hybridizations, in the villages the image of the place remains uncontaminated. In both cases, the identity fulcrum that finds its image in Orthodox religious architecture remains clear, maintaining its individuality as a central meeting place and an identification symbol within the new territorial expansions. With the identification of the distinctive character of the place within the structure of the church, the project developed a first analysis for the definition of a representation protocol that would allow to describe and leave the image of Upper Kama territory unchanged in the historical memory, delivering through the graphic representation an opportunity of connection between territory, city and religious architecture. These connections define a widespread heritage, isolated within the individual territorial realities. The image of the place is represented as a result of a process of synthesis of perceptual memory that the observing subject performs, in an act that Simmel would define as Stimmung [10], where the processing of the serial images that the subject records, by observing a specific

Fig. 5. The relationship between the religious building, still active today, and the territorial context, highlighted in the individual objects that define the complex from the monument and connect to the size of the site. Case study of St Nikolosk church in Nyrob, Cherdyn district.

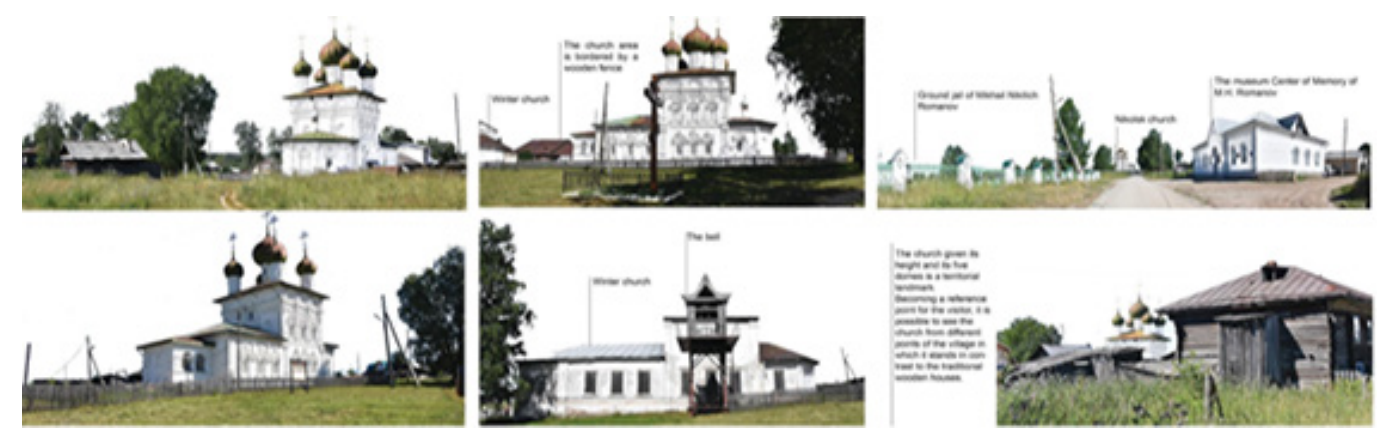


Fig. 6. Digital morpho-metric survey database from terrestrial laser scanner survey The planning of the acquisition has be focused on the adequa focused on the adequacy time in terms of detail, time in terms of detail maintained at the conlevel, and of extension, dedicated both to the complex and to the site.

Fig. 7. Reunified database of the historic center of Solikamsk: the referencing of monuments and of connecting routes allows a better understanding of the planning and service qualities of the territoria system, but it also recalls extensive amounts of data of complex management and manipulation for the analysis.
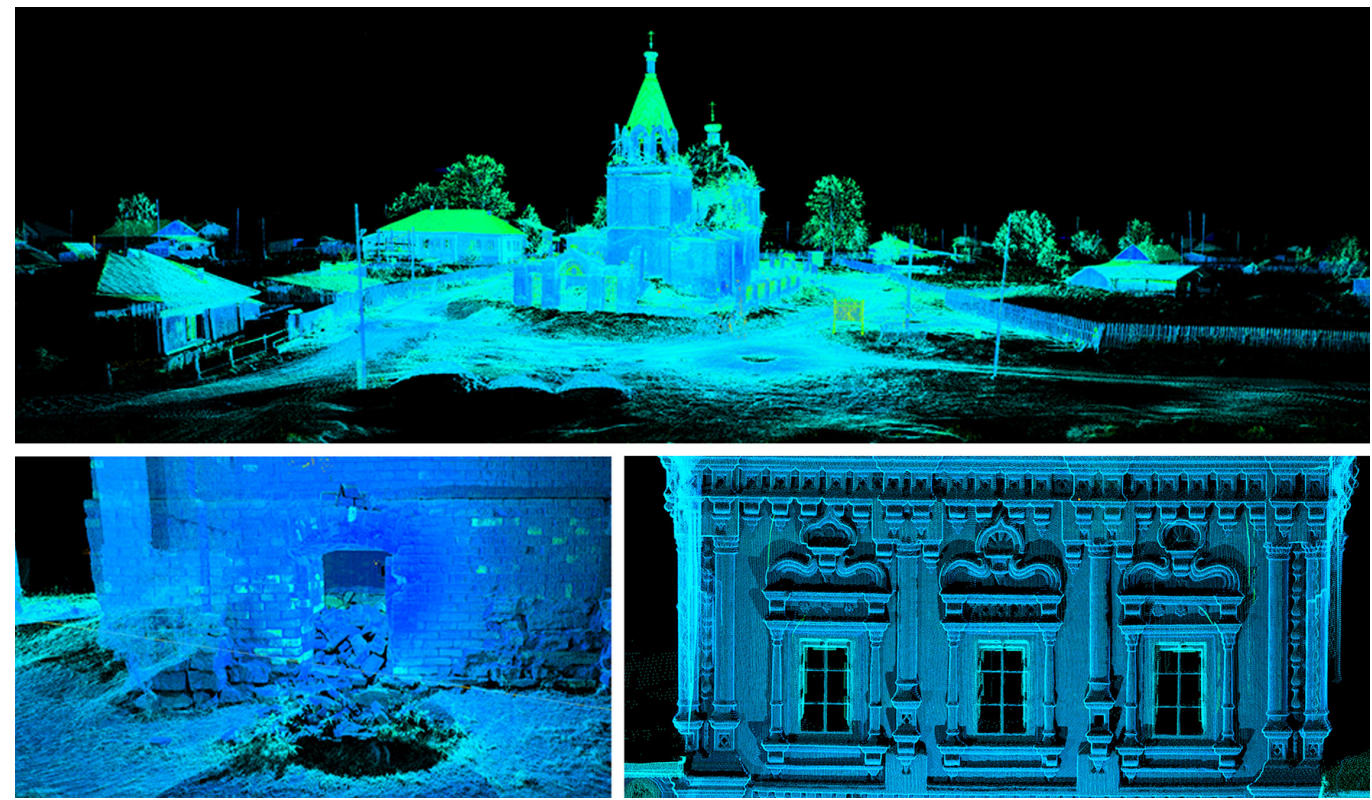

physical space and independently filtering the data, conditioned by his own experiences, draws the representation of a synthetic image within his memory (fig. 5).

During the first year, the project has developed the objective of documenting the conservation status of all the structures present along the Upper Kama route, through their census. Identified and catalogued within a categorized abacus, the eighty religious complexes located between Cherdyn, Usolye and Solikamsk have assumed the operations of an integrated survey of both the single religious complex and the territorial system surrounding them, with the differentiation of acquisition methodologies.

For the territorial system, wide coverage acquisition tools were used, a mobile laser scanner $[\mathrm{II}$ ] for the identification and structuring of territorial and urban interconnection routes, as well as the structuring of flight plans with UAV systems [12] for the restitution of a territorial photogrammetric model to integrate the metric data acquired. At the same time, a photographic campaign was structured aimed both at the acquisition of the territorial macrosystem and at the description of the single religious complex, till to reach the analysis of the detail in the single construction and decorative architectural element, in order to create

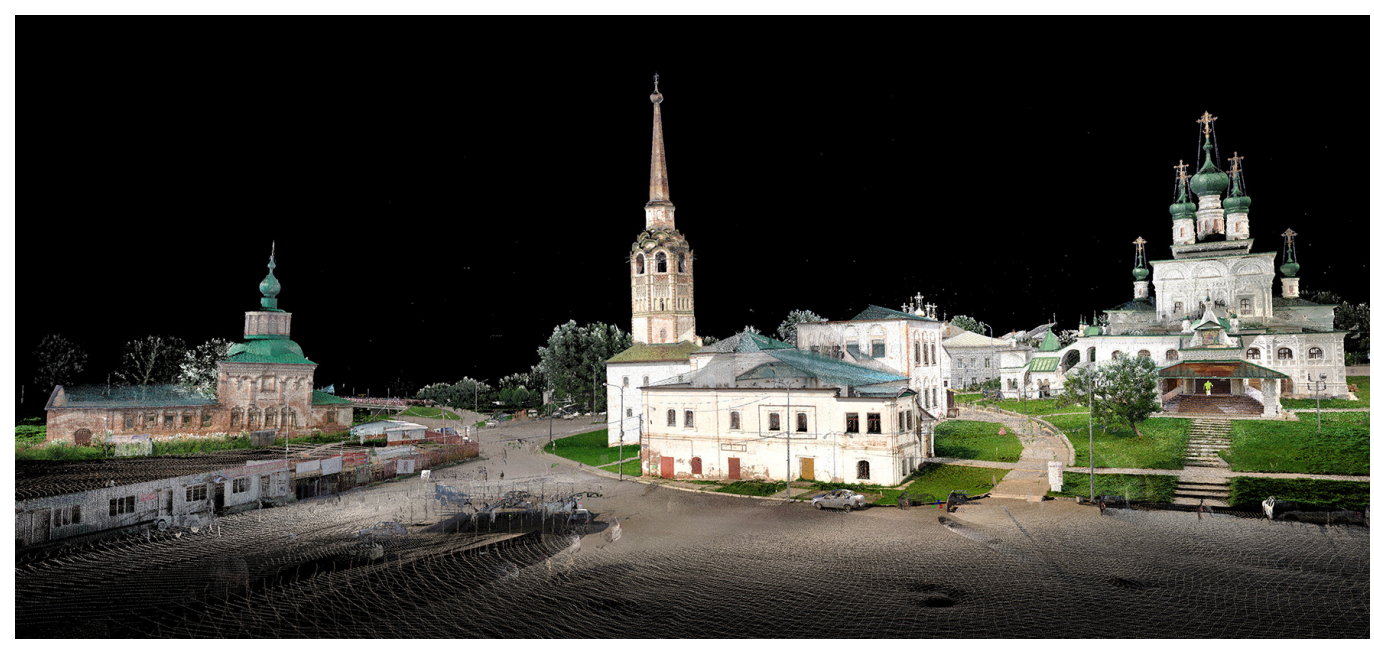


a data library for the definition and the census of the elements characterizing the building. The spatial data was integrated through the structuring of laser scanner surveys (FARO SI 50 series) for the documentation of the architectural detail (fig. 6-9)

The optimization and data collection for the structuring of digital libraries is followed by the information implementation with knowledge on construction technologies and census archives relating to architecture, together with the historical and territorial planning practices, both collected within the compiling of technical sheets used as a basis for the definition of the descriptive criteria necessary for the structuring of a unique digital modelling language (fig. I0).

Fig. 8. Territorial survey strategies applied to Cultural Heritage Routes: aerial camera photogrammetric survey, applied to the urban site and the monument; RG point cloud products, less detailed but more extensive in the storage of spatial and morphometric data.

Fig. 9.Territorial surveying strategies applied to mobile surveying with LiDAR KAARTA: the concept of acquisition trajectory and self-referencing in space supports the documentation of extended systems by concentrating the survey on the connection route as well as on the monument, also contributing to the fast operations on site.

Fig. 10. Identification of the decomposition apparatus of the monument and the route within the census cataloguing sheets, suitable to be compiled on site through iOS app.
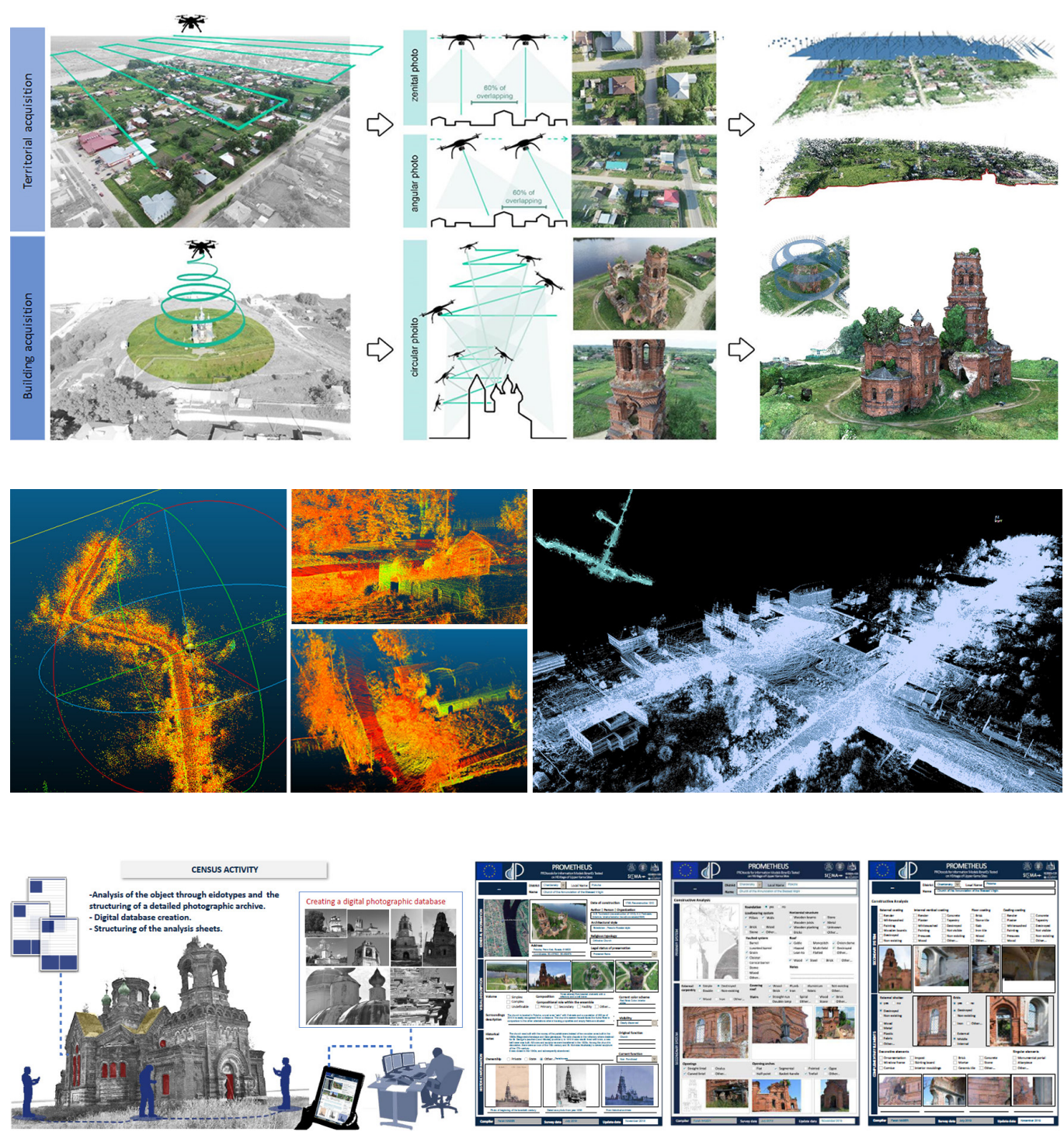

\section{Information models and territorial landscapes}

The theme of the reproducibility of the historical heritage located within a vast territorial system keeps the debate open on the problems of structuring and sharing a type of unified language, consisting of signs and symbols, that can translate the territorial interconnection system through its codification into a generalizable formal scheme. 
Fig. II. The semantic subdivision of the elements, for the study of the categories of conserved elements.

\section{Fig. 12. Complexity} of parameters and connections between the information system project and architectural complexities. First considerations on the construction of geometrically simplified modelling supports, even of high informative content, which can allow the management of descriptive the territory.
Through the discipline of representation, a graphic language is defined in which the complexity of reality is reduced in favour of a selective communication of information. The drawing makes explicit those descriptive needs that animate the form of communication and which therefore operate, through the graphic synthesis, the transmission of information. To this objective, a process of analysis and identification of the semantic anthology of the surveyed buildings, which are part of the widespread heritage of Upper Kama, is necessary to structure a hierarchy for the geometric models, on which to set the management of the information collected during the acquisition phases and archival investigation (fig. I I).

In order to structure an accessible and interoperable information collection platform, this type of methodological approach of Building Information Modeling applied to Cultural Heritage opens a potential range of applications, allowing the flow of data and the possibility of management for the integration at scales higher than the one of the single building, extending from the architectural analysis to the urban, territorial one, and above all to the infrastructural design and programming, a context in which this strategy can represent a paradigmatic change aimed at managing more effectively the complexity of the territorial structure.

In its design phase, the protocol specifies the guidelines to support the modelling, through the structuring of a diversified shared schedule based on the definition of different representation LODs for the detail of the model to differentiate the investigation levels, coding each element in specific model categories.
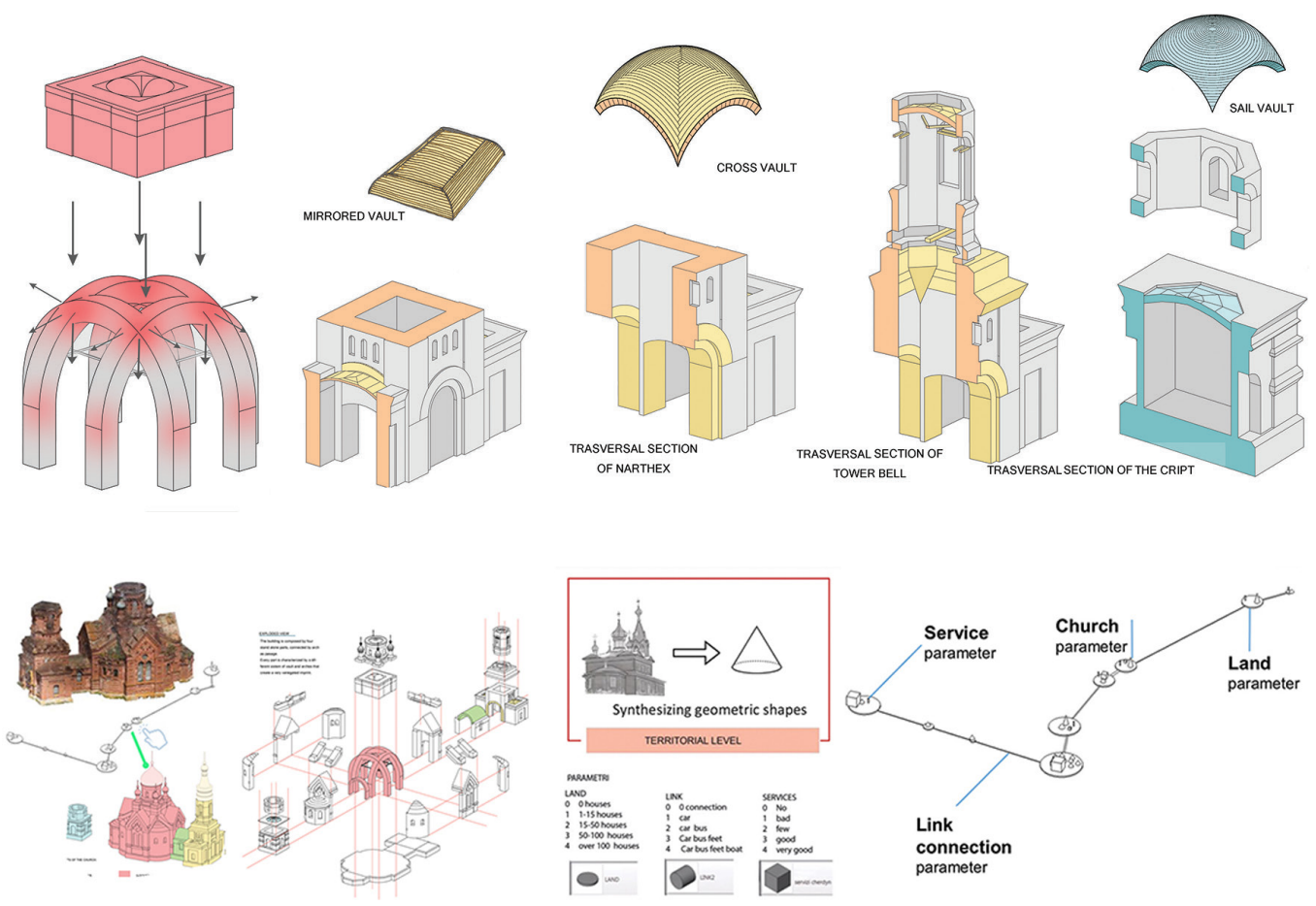

A differentiation in the graphic language of the model is necessary for the preparation of multiple hierarchies of content defined through the use of essential forms, for the structuring of a model system that can be descriptive of the territorial condition.

The definition of model classes, through the structuring of typological abacus of families, allows the systems of interconnection between the architectural complexes and the landscape system to be identified and collected within the three-dimensional space. The model modifies its shape according to the purposes and types of descriptor it assumes in the global system. When it is a descriptor of the territorial system, it will be represented through the association of a volume of synthesis of the geometric form, on the contrary when it is a descriptor of the architectural complex, the geometry, first synthesized, will be implemented 
with the information contents for a detailed reading made available to different intervention figures for architectural and territorial management (fig. I2).

The methodological choice is made with the awareness of the large historical-architectural catalogue of elements that characterize the architecture of Upper Kama sites. Therefore, the investigation operations and the writing of a specific manual for the collection of model components can be useful for structuring data integration processes, through the direct comparison between real objects and virtual models.

\section{Notes}

[I] The editorial responsibility of the contribution is recognized to Raffaella De Marco for paragraphs I and 2, to Anna Dell'Amico for paragraphs 3 and 4.

[2] The idea of 'diffusivity' of Cultural Heritage is now recalled regarding the growing demands for its "accessibility", but it has to be understood not only in the physical terms of the specific site but more generally in the mapping and control of data dispersed in a unified framework of knowledge, monitoring and cultural planning. In this regard, the use of information archives and referencing containers is increasingly required, expanding the influence of visual representation methodologies and updating graphic products in terms of digitization and metadata network [Parrinello, Dell'Amico, 20 I 9, pp. I032- I044].

[3] "Thinking today about the theme of Cultural landscapes [...] makes local and global visions of the contemporary world necessary". Since in the process of economic and cultural globalization the recognition of the identity (and otherness) of a cultural basin presents itself as a crucial problem, the question of the representation of cultural landscapes is linked to that of space and of its processes of symbolization. "In each landscape, therefore, there is the coexistence of the object, its physical extension, its space and the mental representations of a community, in their mutual problematic articulation." The cultural landscape therefore "is not only a pure physical extension to be used in a technical/economic sense but a place where a "cultural mediation" takes place giving shape to the space." The result is "the decisive importance of its representation, not only the mental one, but also of its concrete forms", increasingly encouraged by an intertextual connection capable of recognizing technical-practical dimensions of representative tangibility and geographical extension for the identification of the corresponding apparatus on the territory [Salerno, Casonato 2008, pp. 12-16].

[4] The recognition derives from the report of the meeting of experts of World Heritage Committee WHC-94/CONF.003/ INF. I 3, "Routes as a part of our Cultural Heritage" (Madrid, Spain, November 1994), subsequently implemented by ICOMOS in "Charter on Cultural Routes", 2008. The recognition of the heritage of the routes is mainly intended as "sum of elements referring to a whole [...] within a joint system which enhances their significance" bringing to a "dynamic conformation of cultural landscape". The recent inclusion of most of these routes (for example, Hansa 1991, El Legado Andalusi 1997, the industrial heritage of Rhine Valley 2002, the Pyrenees Route 2003, the Cluniac Sites 2005, the Transromanica 2007, the Cistercian abbeys 2010, the historical thermal towns 2010, the fortresses of Charles V 20I5, the fortified cities of Grande Région 2016) explains why their documentation on the historical and physical conditions, the architectural value and the operational intervention programs for territorial management are still scarce. The existing projects mainly focus on a touristic educational and training approach (HECTOR and CERTESS projects, from the European Institute of Cultural Routes), lacking a dedicated protocol for the unified territorial management of the sites.

[5] The research by A.S. Teryohin, G. D. Kantorovich, I.V. Makovetskiy and V. A. Tsypushtanov made a fundamental contribution to the knowledge of Upper Kama's heritage in their 1970 work. In 1988 the monograph by Kostochkin has integrated previous research, in addition to the archive of the Perm Scientific Restoration Atelier which since 1986 has provided reliable data on existing monuments for the development of conservation and restoration projects. The most recent study on Upper Kama architecture is conducted by W. Brumfield in 2012 in the form of a photographic report.

[6]The documentation of the built heritage of Upper Kama is part of an activity program developed since 20 I 3 by prof. Sandro Parrinello from University of Pavia and prof. Svetlana V. Maximova from Perm National Research Polytechnic University. Three summer schools were organized in 2015,2016 and 2018 involving professors, researchers and students from international universities, and supported by the contribution of the Russian Academy of Painting, Sculpture and Architecture, Stroganov Chambers Historic Architectural Museum in Usolye and by the administration of Usolye.

[7] The Perm Krai Center for Monuments Protection today deals with the Upper Kama heritage for its preservation and the development of intervention and protection projects, such as the case of the Protecting Zone for the historic center of Usolye.

[8] Following the Soviet rise, many complexes were abandoned or saw a conversion of their spaces for other functions, mainly for energy or food production (such as the Church of the Annunciation of the Blessed Virgin of Pokcha converted into an electrical power central in 1940, the case of the Church of the Exaltation of the Holy Cross in Solikamsk, abandoned in 1929 and later transformed into a brewery, and the Church of the Epiphany in Cherdyn, now occupied by a bakery). The central event is represented by the construction of the electricity station on the Kama in 1954, with the flooding of the river and the variation of the territorial hydrogeological relationship between the groundwater levels and the foundation sites of the complexes, the cause of obvious collapses and instability conditions which distinguish today the condition of emergency assets.

[9] PROMETHEUS project "PROtocols for information Models librariEs Tested on Heritage of Upper Kama Sites" collects and develops such research within the European funding program Horizon 2020-R\&I-RISE- Research \& Innovation Staff Exchange Marie Skłodowska-Curie. The project (coordinated by Prof. S. Parrinello from University of Pavia) involves the collaboration between three international universities (University of Pavia, Universitat Politecnica de Valencia, Perm National Research Polytechnic University) and two companies (SISMA srl, EBIME sl.). The project includes the participation of | I Experienced Researchers and 5 Early-Stage Researchers, as well as ordinary and master students in engineering and architecture from the involved universities. This project has received funding from the European Union's Horizon 2020 research and innovation programme under the Marie Skłodowska-Curie grant agreement No 821870.

[I0] "The landscape, we can say, is created when the various natural elements lying close to each other on the earth's soil come together in a very particular kind of unit, different from that glimpsed by the scientist with his causal thought, by the 
nature worshiper with his religious sentiment, by the farmer or strategist with their theological approach." Simmel defines Stimmung, a quality of the landscape as a human feeling. Without this feeling, the landscape would be only a sum of natural elements. This however is not performed since the man, while contemplating it, has an attitude of elaboration, in the sense that he perceives the elements of nature and unifies them. Making them his own, he absorbs the substance given by nature and re-elaborates it through the act of contemplation [Simmel 1985]

[I I] The KAARTA Stencil mobile laser scanner bases its data acquisition system on a reworking of LIDAR and IMU data for localization. The system uses Velodyne (VLP-I 6) connected to a low-cost IMU MEMS and a processing computer for real-time mapping and localization. The KAARTA and FARO instruments are made available by PLAY laboratory of the Department of Civil Engineering and Architecture of University of Pavia.

[12] For the territorial acquisition for photogrammetric aerial processing, a DJl Phantom 4 Pro series drone was used, made available by DAda Lab of the Department of Civil Engineering and Architecture of University of Pavia.

\section{References}

Brumfield William Craft (1993). A history of Russian architecture. Cambridge: University press, pp. 664.

Brumfield William Craft (2008). The architectural heritage of Solikamsk and the Northern Districts of Perm Province. In Cahiers slaves. Routes et Chemins slaves, sous la direction de Laure Troubetzkoy et Fracis Conte, I0, pp. 317-355.

Di Giulio Roberto et al. (2017). Integrated data capturing requirements for 3D semantic modelling of cultural heritage: the inception protocol. In ISPRS - International Archives of the Photogrammetry, Remote Sensing and Spatial Information Sciences. XLII2M3. 25 I-257. 10.5194/isprs-archives-XLII-2-W3-251-2017.

Jackob Michael (2019). II paesaggio. Bologna: II Mulino.

Jordan Isabel et al. (20।8). Protocol to Manage Heritage-Building Interventions Using Heritage Building Information Modelling (HBIM). Sustainability. 10. 908. 10.3390/su 10040908.

Kioussi Anastasia et al. (2012). Integrated documentation protocols enabling decision making in cultural heritage protection. In Journal of Cultural Heritage, I 4 (3), pp. I4I-146.

Kostochkin V.V., (1988). Cherdyn, Solikamsk, Usolye. Moscow: Stroiizdat.

Parrinello Sandro et al. (2019). Documenting the Cultural Heritage Routes. The creation of informative models of historical Russian churches on Upper Kama Region. In Int. Arch. Photogramm. Remote Sens. Spatial Inf. Sci., XLII-2M I 5, 2019, pp. $887-894$.

Parrinello Sandro, De Marco Raffaella (2019. Integration and modelling of 3D data as strategy for the structural diagnosis in Endangered Sites. The study case of Church of the Annunciation in Pokcha (Russia). In Proceedings of 2019 IMEKO TC-4 International Conference on Metrology for Archaeology and Cultural Heritage. Firenze: IMEKO, pp. 223-228.

Parrinello Sandro, Dell'Amico Anna (2019). Experience of Documentation for the Accessibility of Widespread Cultural Heritage. In Heritage, 2( I), pp. 1032- 1044.

Parrinello Sandro, Cioli Federico (2020). Establishment of a complex database for the study of Cultural Heritage through the reading and analysis of the traditional architecture of Upper Kama. In Kremers Horst. In Digital Cultural Heritage. Cham: Springer, pp. 5|-6|.

Parrinello Sandro, Cioli Federico (20 I 8). Un progetto di recupero per il complesso monumentale di Usolye nella regione della Kama Superiore. In Restauro Archeologico, I, 2018, pp. 92-1 II.

Parrinello Sandro, Maksimova Svetlana, Mezenina Kseniia (20I5). Historic environment architectural survey with the use of digital technology. Vestnik Permskogo Nacional'nogo Issledovatel'skogo Politehničeskogo Universiteta. In Urbanistika, I, pp. $102-117$.

Parrinello Sandro, Maksimova Svetlana V. (2016). Contemporary methods of urban environment architectural survey. Perm: Polytechnic University.

Parrinello Sandro, Picchio Francesca (20I4). Recomposing the landscape using structure from motion survey in the village of Korza, Karelia (Russia). In SCIRES, 4, 2, pp. 29- 42.

Simmel George (1985). Philosophie der Landschaft, in "Die Güldenkammer", I 9 I 3. (Trad. it. Filosofia del paesaggio, in Id., II volto e il ritratto. Saggi sull'arte, Bologna: il Mulino.

Teryohin A.S. et al. (1970). Monuments of stone architecture of XVI-XVIII in Solikamsk. Perm.

\section{Authors}

Raffaella De Marco, Università di Pavia, raffaella.demarco@unipv.it

Anna Dell'Amico, Università di Pavia, anna.dellamico@unipv.it

To cite this chapter. De Marco Raffaella, Dell'Amico anna (2020). Connettere il territorio tra patrimonio e informazione: banche dati e modelli per le Cultural Heritage Routes/Connecting the territory between Heritage and Information: databases and models for the Cultural Heritage Routes. In Arena A., Arena M., Brandolino R.G., Colistra D., Ginex G., Mediati D., Nucifora S., Raffa P. (a cura di). Connettere. Un disegno per annodare e tessere. Atti del $42^{\circ}$ Convegno Internazionale dei Docenti delle Discipline della Rappresentazione/Connecting. Drawing for weaving relationships. Proceedings of the 42th International Conference of Representation Disciplines Teachers. Milano: FrancoAngeli, pp. 2058-2077. 